\title{
Necrosome complex detected in granulovacuolar degeneration is associated with neuronal loss in Alzheimer's disease
}

Marta J. Koper ${ }^{1,2,3}$, Evelien Van Schoor ${ }^{1,3,4}$, Simona Ospitalieri ${ }^{1}$, Rik Vandenberghe ${ }^{5}$, Mathieu Vandenbulcke $^{6}$, Christine A.F. von Arnim ${ }^{7,8}$, Thomas Tousseyn ${ }^{9}$, Sriram Balusu, ${ }^{2,3,+}$, Bart De

Strooper ${ }^{2,3,10+*}$, Dietmar Rudolf Thal ${ }^{1,9+*}$

1. Laboratory for Neuropathology, Department of Imaging and Pathology, KU Leuven (University of Leuven), Leuven Brain Institute (LBI), Leuven, Belgium

2. Laboratory for the Research of Neurodegenerative Diseases, Department of Neurosciences, KU Leuven (University of Leuven), Leuven Brain Institute (LBI), Leuven, Belgium

3. Center for Brain \& Disease Research, VIB, Leuven, Belgium

4. Laboratory for Neurobiology, Department of Neurosciences, KU Leuven (University of Leuven), Leuven Brain Institute (LBI), Leuven, Belgium

5. Department of Neurosciences, Experimental Neurology Group, KU Leuven and Department of Neurology, UZ Leuven, Leuven, Belgium

6. Department of Geriatric Psychiatry, University Hospitals Leuven, Leuven, Belgium

7. Department of Neurology, University of UIm, Ulm, Germany

8. Department of Geriatrics, University Medical Center Göttingen, Göttingen, Germany

9. Department of Pathology, University Hospital Leuven, Leuven, Belgium

10. UK Dementia Research Institute at University College London, London, UK

+ these authors contributed equally and are listed in alphabetical order

* Corresponding authors:

Prof. Bart De Strooper

Laboratory for the Research of Neurodegenerative Diseases

Department of Neurosciences

VIB-KU Leuven Center for Brain \& Disease Research

O\&N IV Herestraat 49, box 602

3000 Leuven

Belgium

Tel: +32 16373246

E-mail: bart.destrooper@kuleuven.vib.be

Prof. Dietmar R. Thal

Laboratory for Neuropathology

Department of Imaging and Pathology

O\&N IV, Herestraat 49, box 1032

3000 Leuven

Belgium

Tel: +32 16344047

E-mail: dietmar.thal@kuleuven.be 


\begin{abstract}
Alzheimer's disease $(A D)$ is characterized by a specific pattern of neuropathological changes, including extracellular amyloid $\beta(A \beta)$ deposits, intracellular neurofibrillary tangles (NFTs), granulovacuolar degeneration (GVD) representing cytoplasmic vacuolar lesions, synapse dysfunction and neuronal loss. Necroptosis, a programmed form of necrosis characterized by the assembly of the necrosome complex composed of phosphorylated proteins, i.e. receptorinteracting serine/threonine- protein kinase 1 and 3 (pRIPK1 and pRIPK3), and mixed lineage kinase domain-like protein ( $\mathrm{PMLKL}$ ), has recently been shown to be involved in AD. However, it is not yet clear whether necrosome assembly takes place in brain regions showing ADrelated neuronal loss, and whether it is associated with AD-related neuropathological changes. Here, we analyzed brains of $A D$, pathologically defined preclinical $A D$ (p-preAD), and non-AD control cases to determine the neuropathological characteristics and distribution pattern of the necrosome components. We demonstrated that all three activated necrosome components can be detected in GVD lesions (GVDn+, i.e. GVD with activated necrosome) in neurons, that they colocalize with classical GVD markers, such as pTDP-43 and CK1ס, and similarly to these markers detect GVD lesions. GVDn+ neurons inversely correlated with neuronal density in the early affected CA1 region of the hippocampus and in the late affected frontal cortex layer III. Additionally, AD-related GVD lesions were associated with AD-defining parameters, showing the strongest correlation and partial colocalization with NFT pathology. Therefore, we conclude that the presence of the necrosome in GVD plays a role in $A D$, possibly by representing an AD-specific form of necroptosis-related neuron death. Hence, necroptosis-related neuron loss could be an interesting therapeutic target for treating AD.
\end{abstract}

\title{
Keywords
}

Necroptosis, granulovacuolar degeneration, Alzheimer's disease, neuronal loss, necrosome, pMLKL 


\section{Introduction}

Alzheimer's disease $(A D)$ is one of the most common forms of dementia, accounting for $60 \%$ to $80 \%$ of the demented individuals and affecting 48 million people worldwide [1]. The clinical manifestation of $A D$, characterized by memory loss and cognitive decline, is preceded by neuropathological changes leading to progressive functional and structural degeneration [48, 72]. Accumulation of misfolded proteins, associated synaptic dysfunction and neuronal loss are considered as the most important hallmarks of neurodegenerative diseases, including AD. The extensive extracellular deposition of amyloid $\beta(A \beta)$ protein in plaques and the intracellular formation of neurofibrillary tangles (NFTs), composed of hyperphosphorylated tau (pTau) protein, constitute AD-specific processes [9]. These changes are associated with a neuroinflammatory reaction, gliosis, neurovascular alterations [4, 85], and loss of synapses and neurons [61], leading to cerebral atrophy [24, 27, 54].

Another lesion observed in AD brains is granulovacuolar degeneration (GVD). GVD inclusions are membrane-bound intraneuronal vacuoles (3-5 $\mu \mathrm{m}$ diameter) with a dense argyrophillic core [26], and are considered to be pathological structures originating from the macroautophagic pathway due to their similarity in ultrastructural morphology $[35,53]$. The autophagy-lysosome system is one of the major routes for protein degradation in cells, and dysfunction in protein clearance was shown to contribute to AD pathogenesis [17]. For instance, alterations in lysosomal degradation of Tau were associated with increased formation of oligomeric Tau and neurotoxicity [77]. In turn, resulting lysosomal dysfunction may cause a shift towards the development of GVD granules, which are believed to be late-stage autophagic organelles, according to some authors [18, 80]. Evidence suggests that GVD lesions are cytoplasmic inclusions sequestering post-translationally modified and often toxic proteins, including proteins related to AD pathology [35], such as pTau [15] and phosphorylated $A \beta$ (Ser26) [37]. Therefore, GVD granules accumulating aberrant, nondegraded proteins [35] may be a consequence of upstream processes induced by abnormal protein aggregates, such as pTau and $A \beta$, which affect lysosomal function. However, the molecular composition and biochemical properties of these granules are not known [35]. GVD pathology has been shown to be strongly associated with degenerating neurons in $A D[2,64$, 74], but it can also be present to a lesser extent in other neurodegenerative diseases and in the aging brain [3, 57, 75, 81]. GVD primarily affects hippocampal pyramidal neurons [2], but as $A D$ progresses, a systematic spreading of GVD lesions along anatomically connected regions has been observed [74]. Interestingly, GVD-positive cells show considerable topographic overlap with abnormal pTau accumulation, initiating in related regions, i.e. the hippocampus and neighboring entorhinal cortex $[2,15]$. Additionally, tau-seeds have recently been shown to induce the formation of granulovacuolar bodies in an in vitro model [80]. 
Nevertheless, given the conflicting evidence on the relationship between GVD and pTau pathology, their individual and/or dependent effect on the pathological cascade of AD is not yet clear $[28,34,38,65]$.

Although the majority of studies in the field of $A D$ are focused on $A \beta$ and $p T a u$, there is a lack of knowledge about the mechanisms underlying neuronal degeneration and their relation to $A D$-associated lesions. The hippocampal formation is a key region for $A D$ pathology $[9,16,29$, 71], and its degeneration is widely used in clinical practice as a diagnostic marker. In contrast to $A \beta$ pathology, which is present in neocortical areas before the hippocampus gets affected [72], GVD and NFTs are more pronounced in medial temporal regions [2, 15]. Given that neuronal loss also initiates and is most prominent in medial temporal areas, the mechanism underpinning $A \beta$-related neuronal loss does not seem to be directly linked with cell death mechanisms specific for pTau or GVD pathology. Earlier studies indicated the role of apoptosis, a programmed form of cell death, in AD-associated neurodegeneration. Nevertheless, apoptotic neurons are rarely detected in $A D$ brains $[65,66]$. Recently, necroptosis has been shown to be activated in amyotrophic lateral sclerosis (ALS) [31, 55], multiple sclerosis (MS) [50] and AD [10]. This caspase-independent programmed form of necrosis is executed by a phosphorylated form of mixed lineage kinase-like pseudokinase (pMLKL), which oligomerizes upon phosphorylation by receptor-interacting serine/threonineprotein kinase-3 (RIPK3) [23, 68, 83, 84]. In turn, pRIPK3 is controlled by pRIPK1, which as a non-activated protein is part of complex-I of the tumor necrosis factor receptor-1 (TNFR1) signaling pathway [13]. pRIPK1-pRIPK3 necrosome assembly is required for MLKL recruitment, phosphorylation-mediated activation and translocation to the plasma membrane to execute necroptosis [12]. The necroptotic pathway can be induced by viral, bacterial or chemical exposure, radiation or endogenous factors [22], and cellular death is usually observed a few hours after activation of the pathway [58]. This type of programed cell death is characterized by morphological changes, including swelling of cells and organelles, increased cytoplasmic granularity, loss of membrane integrity and expulsion of cellular contents, which induces significant inflammation [22].

While cell death is compelling in AD pathology, it remains unclear which factors contribute to neuronal degeneration and how exactly this process is executed. To analyze necroptotic cell death in the $A D$ brain, we characterized the neuroanatomical distribution of the necrosome components ( $p$ RIPK1, pRIPK3 and pMLKL) and their relation to AD-specific neuropathological changes. Moreover, we also studied non-phosphorylated forms of these proteins as they have previously been shown to be involved in AD-associated necroptosis [10]. We used postmortem brain tissue from non-demented elderly, pathologically diagnosed preclinical AD ( $p$ preAD) and symptomatic $A D$ cases. The group of $p$-preAD cases covered non-demented 
individuals that showed low-high degrees of $A D$ neuropathological changes as defined according to the recommendations of the National Institute on Aging and the Alzheimer's Association (NIA-AA) at autopsy [30, 56]. Accordingly, we report for the first time the relation between necrosome components and GVD in the context of AD-related neuronal loss, possibly indicating GVD granules as a potential protective system trapping the necrosome complex, which could interfere with or delay neuronal death.

\section{Material and methods}

\section{Neuropathology}

Brain tissue samples from $23 \mathrm{AD}, 24$ p-preAD, and 16 non-demented control patients were included in this study (Table 1). The autopsies were performed with informed consent in accordance with the applicable laws in Belgium (UZ Leuven) and Germany (Ulm, Bonn, and Offenbach). The use of human tissue samples for this study was approved by the UZ Leuven ethical committee (Leuven, Belgium). Brain tissues were collected with an average postmortem interval (PMI) of 39 hours. After autopsy, the right hemisphere including brainstem and cerebellum was dissected for gross neuropathological assessment and then stored at $-80^{\circ} \mathrm{C}$. The left hemisphere including brainstem and cerebellum was fixed in $4 \%$ phosphate buffered formaldehyde for two-four weeks and dissected for gross neuropathological assessment. Blocks from frontal, parietal, and occipital lobe (Area 17), cingulate gyrus, hippocampus with temporal cortex, entorhinal cortex, hypothalamus, basal ganglia, amygdala, basal nucleus of Meynert, thalamus, midbrain, pons, medulla oblongata and cerebellum were taken, dehydrated, and embedded in paraffin. Five $\mu \mathrm{m}$ sections were cut using an electronic rotary microtome (Thermo Fisher Scientific), mounted on Flex IHC adhesive microscope slides (Dako) and dried at $55^{\circ} \mathrm{C}$ before storing. For neuropathological analysis, sections from all blocks were stained for hematoxylin-eosin, pTau (AT8), A (4G8), phosphorylated transactive response DNA binding protein 43 kDa (pTDP-43), and casein kinase (CK) $1 \delta$ (Suppl. Table 1). The post-mortem diagnosis of $A D$ pathology was based upon the standardized clinicopathological criteria, including the topographical distribution of $A \beta$ plaques in the medial temporal lobe (ABMTL phase) based on $A \beta$ immunohistochemistry [73], the Braak neurofibrillary tangle (NFT) stage based on pTau immunohistochemistry [8, 9], the Consortium to Establish a Registry for Alzheimer's disease (CERAD) score for neuritic plaque frequency [44], the NIA-AA degree of AD pathology [30], and the stage of GVD pathology [74]. To determine the stage of cognitive and functional impairment, the Clinical Dementia Rating (CDR) score [45] was retrospectively assessed in 60 cases, as previously described [24]. The study comprised 63 cases with an average age of 71 (range 18 to 98) years, and a female to male ratio of $25: 38$. The cases were divided in three groups based on the clinical and 
neuropathological diagnosis: 1) $A D=$ intermediate or high NIA-AA degree of $A D$ pathology and signs of cognitive decline during life (CDR $\geq 0.5) ; 2)$ p-preAD = cases with low - high NIA-AA degrees of $A D$ pathology lacking clinical signs of cognitive decline $(C D R=0)$; 3 ) non-AD = no pathological signs of $A D$ pathology (NIA-AA degree $=0$ ). Primary age-related tauopathy (PART) [14] was present in one non-AD control. The clinicopathological characteristics of the identified cases are summarized in Table 1.

\section{Immunohistochemistry}

The expression and distribution of RIPK1, pRIPK1, RIPK3, pRIPK3 and pMLKL were examined in human samples of the hippocampus, entorhinal cortex and temporal cortex using immunohistochemical techniques. The primary antibodies with appropriate dilutions and pretreatments are summarized in Suppl. Table 1. For pRIPK1, pRIPK3, and pMLKL, the samples of the hypothalamus, amygdala and frontal cortex were additionally analyzed. Briefly, paraffin sections were deparaffinized in a robotized autostainer (Leica Microsystems) and pretreated with low ( $\mathrm{pH}$ 6.1) or high $\mathrm{pH}(\mathrm{pH}$ 9) buffer using a PT Link module (Dako). After incubation with Envision Flex Peroxidase-Blocking Reagent (Dako) for $5 \mathrm{~min}$, the samples were blocked with $5 \%$ bovine serum albumin (BSA) for $30 \mathrm{~min}$. Then, the tissues were incubated in a humid chamber overnight at room temperature (RT) with the primary antibodies, followed by incubation with HRP-conjugated secondary antibodies (Dako) for 30 min. 3,3'diaminobenzidine solution (Liquid DAB+ Substrate Chromogen System, Dako) was used as a chromogen to yield brown reaction products. Counterstaining with hematoxylin and dehydration were carried out in the autostainer, followed by mounting in an automated coverslipper (Leica Microsystems). Microscopy analysis was performed using a light Leica DM2000 LED microscope (Leica Microsystems) and images were captured with a Leica DFC7000 T camera (Leica Microsystems).

Specific binding of antibodies against pRIPK1, pRIPK3, and pMLKL was confirmed by two separate methods. First, to determine whether the antibodies specifically bind to their target proteins, the respective antibodies were preincubated with their specific blocking peptides; i.e. the peptides against which they were raised (antibody to blocking peptide ratio 1:5 for pMLKL, Abcam; 1:2 for pRIPK1 and pRIPK3, Cell Signaling). Afterwards, the mix was used for immunostaining. In parallel, we performed the respective antibody stainings without blocking peptides. The absence of a staining signal obtained in the presence of the blocking peptide was considered to confirm specific binding. Secondly, an alkaline phosphatase dephosphorylation assay on paraffin-embedded hippocampal sections was used to examine specific binding of the antibodies to the phosphorylated epitopes and the detection of the nonphosphorylated proteins after dephosphorylation. The latter was possible only for RIPK1 and RIPK3 because the MLKL-antibody did not show proper staining in immunohistochemistry. 
Tissue sections of the medial temporal lobe were incubated with a solution composed of alkaline phosphatase (Sigma-Aldrich) diluted in Tris buffer with phenylmethylsulfonyl fluoride (Sigma-Aldrich; 1:20 for pMLKL and pRIPK3, 1:5 for pRIPK1), for $24 \mathrm{~h}$ at $30^{\circ} \mathrm{C}$ (pMLKL and pRIPK3) or for 4 days at $30^{\circ} \mathrm{C}$ (pRIPK1; the solution was refreshed every $24 \mathrm{~h}$ ). Then, immunohistochemistry for the phosphorylated markers (pRIPK1, pRIPK3 and pMLKL) and non-phosphorylated proteins (RIPK1 and RIPK3) was performed. In parallel, untreated (nondephosphorylated) sections were reacted with the same antibodies. For these experiments, the same protocol for immunohistochemistry was followed as described above.

\section{Immunofluorescence}

Colocalization of the proteins of interest was investigated by multiple labeling immunofluorescence. Sections from the medial temporal lobe (MTL) were pre-treated as mentioned above, and incubated with formic acid for $3 \mathrm{~min}$, when required. Multiple immunostainings were performed either with an antibody cocktail or sequentially (when using two or more antibodies raised in the same species). In general, primary antibodies against proteins of interest (Suppl. Table 1) were detected with species-specific fluorescentconjugated secondary antibodies (Abcam, Jackson ImmunoResearch). The multiple rabbit-onrabbit or mouse-on-mouse staining protocols were optimized based on previously described methods [21]. Briefly, a coupling method was used to avoid cross-reactivity of secondary antibodies when using primary antibodies from the same species. The first rabbit/mouse primary antibody was used as described above, followed by a fluorescent goat antirabbit/mouse secondary antibody. The second rabbit/mouse primary was coupled to a donkey anti-rabbit/mouse Fab fragment (Jackson ImmunoResearch) conjugated to a fluorophore (Jackson ImmunoResearch). For coupling, we incubated the primary antibodies with the respective Fab fragments for 20 min at RT ( $2 \mu \mathrm{g}$ Fab fragment per $1 \mu \mathrm{g}$ primary antibody). Next, normal rabbit/mouse serum (Jackson ImmunoResearch) was added to capture the unbound Fab fragments ( $10 \mu \mathrm{l}$ of serum per $1 \mu \mathrm{g}$ Fab fragment) for another $10 \mathrm{~min}$. Thereafter, these conjugated primary antibodies were used to stain the second epitope. Sections were mounted with an aqueous mounting medium containing DAPI (Invitrogen) for counterstaining of the nuclei. All immunofluorescence images were captured via Nikon NIS-Elements software using a Nikon A1R laser scanning confocal system coupled to a Nikon Eclipse Ti inverted microscope (Nikon Instruments, Inc.). Acquired data were further processed using ImageJ software (National Institutes of Health). All antibodies used for immunohistochemistry and immunofluorescence assays are summarized in Suppl. Table 1. 


\section{Characterization of anti-pMLKL as marker for GVD}

To determine whether anti-pMLKL detects GVD lesions similarly as standard markers for GVD, stainings with the anti-pMLKL antibody were compared to those with the established GVD markers, such as pTDP-43 [33, 74] and CK1ס [62]. Specifically, double labeling immunofluorescence was carried out in six regions of the medial temporal lobe of 12 cases, including CA1, CA2/3, CA4, subiculum, dentate gyrus and entorhinal cortex. A semiquantitative analysis to compare anti-pMLKL and anti-pTDP-43-stained GVD lesions in the medial temporal lobe was performed in 31 cases, which represented the following stages of GVD: stage zero $(n=11)$, stage I $(n=2)$, stage II $(n=0)$, stage III $(n=3)$, stage IV $(n=7)$, and stage $V(n=8)$. To evaluate GVD scores, a 4-point scale $(0-3)$ was used. A score of zero represented no GVD expression ( $0 \%$ positive neurons), a score of one represented mild expression ( $1-25 \%$ positive neurons), a score of two represented moderate expression (26 - $40 \%$ positive neurons), and a score of three represented extensive expression (>40\% positive neurons) in terms of number of affected neurons. GVD scores were calculated for each region of interest (CA1, CA2/3, CA4, subiculum, dentate gyrus and entorhinal cortex) and compared between anti-pMLKL and anti-pTDP-43 for AD and p-preAD cases. To determine differences across the three groups, we compared GVD severity, which was determined for each case as the mean of the GVD scores of the evaluated MTL regions (CA1, CA2/3, CA4, subiculum, dentate gyrus and entorhinal cortex).

\section{Quantitative analysis of GVD-positive neurons and neuronal density in the hippocampal CA1 and the frontal cortex layer III}

For quantification of pMLKL-immunoreactive GVD containing (GVDn+) neurons and total neuronal density in the CA1 region of the hippocampus, three consecutive images $(0,632 \times$ $0,474 \mathrm{~mm}$ ) of anti-pMLKL stained sections were taken using the Leica DM2000 LED microscope with a $20 \times$ objective lens. Similarly, for quantification in layer III of the frontal cortex, five consecutive images were captured. The criteria regarding morphological conditions of cells to be included in the measurement were determined before the quantification. Neurons were identified based on the nuclear pattern of the counterstaining, and GVD granules were detected by pMLKL-immunoreactivity. For both regions, the same analysis was carried out using anti-CK1ס as established GVD marker to examine whether GVD detection with antipMLKL shows equal results compared to anti-CK1ס, especially for the relationship between GVD and neuron loss. Image analysis was performed using ImageJ software and both GVDpositive and unaffected neurons were manually counted in hippocampal CA1 and frontal cortex layer III. The estimates of pyramidal neuron density and the percentages of GVD-containing 
neurons to the total number of neurons were determined separately for GVDn+ neurons detected with anti-pMLKL and neurons bearing CK1ס-positive GVD granules.

\section{Clear, unobstructed brain imaging cocktails and computational analysis (CUBIC)-based clearing and 3D imaging of GVD and NFTs}

Clearing of temporal cortex tissue was performed using the CUBIC method as previously described [69], with minor modifications. In short, the formaldehyde-fixed specimens were sectioned into $1 \mathrm{~mm}$ thick samples with a Leica VT1000S vibratome and washed overnight at RT using phosphate-buffered saline (PBS, $135 \mathrm{mM} \mathrm{NaCl}, 2.7 \mathrm{mM} \mathrm{KCl}, 11 \mathrm{mM}$ Phosphate Buffer, pH 7.5). The tissue sample of one AD case (Case 3, see Table 1) was incubated with CUBIC Reagent-1, composed of Quadrol (Sigma-Aldrich), urea (Sigma-Aldrich) and Triton X100 (Sigma-Aldrich) in $\mathrm{dH}_{2} \mathrm{O}\left(1: 1 \mathrm{v} / \mathrm{v}\right.$ mixture of $\mathrm{dH}_{2} \mathrm{O}:$ Reagent- 1$)$ for $6 \mathrm{~h}$ at $37^{\circ} \mathrm{C}$, and further immersed in CUBIC Reagent-1 overnight and subsequently for 6 days at $37^{\circ} \mathrm{C}$ (Reagent- 1 was refreshed every 2 days). A washing step was performed three times for $2 \mathrm{~h}$ at RT using PBS containing $0.01 \%$ sodium azide. After incubation with a cocktail of pMLKL and AT8 primary antibodies for three days at $37^{\circ} \mathrm{C}$, the samples were washed overnight at $37^{\circ} \mathrm{C}$ with PBS $0.01 \%$ Triton $\mathrm{X}-100$, then washed twice for $2 \mathrm{~h}$ at RT using PBS with $0.01 \%$ sodium azide. The tissues were probed with Cy5-labeled and Cy3-labeled species-specific secondary antibodies (Jackson Immuno-Research) together for four days at $37^{\circ} \mathrm{C}$. Subsequently, they were washed twice for $2 \mathrm{~h}$ at $37^{\circ} \mathrm{C}$ with PBS $0.01 \%$ Triton $\mathrm{X}-100$, and twice for $1 \mathrm{~h}$ at $37^{\circ} \mathrm{C}$ with PBS with $0.01 \%$ sodium azide. Finally, the samples were immersed in CUBIC Reagent-2, comprised of urea (Sigma-Aldrich), sucrose (Sigma-Aldrich) and triethanolamine (Sigma-Aldrich) in $\mathrm{dH}_{2} \mathrm{O}$ ( $1: 1 \mathrm{v} / \mathrm{v}$ mixture of PBS: Reagent-2) for one day at $37^{\circ} \mathrm{C}$, followed by incubation with CUBIC Reagent- 2 for two days at $37^{\circ} \mathrm{C}$, and for one day at RT. Cleared tissues were immersed in a mixture $(1: 1, v / v)$ of silicon oil (Sigma-Aldrich) and mineral oil (Sigma-Aldrich) for image acquisition. Images were captured using a spinning disk confocal microscope (Nikon Instruments, Inc.) with a $20 \times$ objective lens, and processed in Imaris software (Bitplane).

\section{Cell culture and treatment}

SH-SY5Y cells were stimulated for necroptosis and used throughout the study as a positive control for the changes observed in necroptosis-related proteins in human tissue. Briefly, human neuroblastoma SH-SY5Y cells were cultured in DMEM/F12 medium (Thermo Fisher Scientific) supplemented with Penicillin-Streptomycin (Thermo Fisher Scientific). The cells were maintained at $37^{\circ} \mathrm{C}$ in a $\mathrm{CO}_{2}$ incubator with a controlled humidified atmosphere composed of $95 \%$ air and $5 \% \mathrm{CO}_{2}$. For induction of necroptosis, SH-SY5Y cells were stimulated for $4 \mathrm{~h}$ at $37^{\circ} \mathrm{C}$ with TNFa $(30 \mathrm{ng} / \mathrm{ml}$, Sigma-Aldrich) plus SMAC-mimetic $(10 \mu \mathrm{M}$, Sellekchem) plus zVAD-fmk (20 $\mu \mathrm{M}$, Millipore). For collecting, cells were washed with PBS and 
lysed in RIPA buffer (Sigma-Aldrich) containing PhosSTOP (Sigma-Aldrich) and a protease inhibitor cocktail (Sigma-Aldrich). Cell lysates were used for validation of antibodies and as a positive control for western blot. Experiments with the human SH-SY5Y cell line were approved by the UZ-Leuven ethical committee.

\section{Western blot}

Frozen temporal cortex tissues from $A D(n=5)$, preclinical $A D(n=5)$, and non-AD $(n=5)$ patients were homogenized in extraction buffer (2\% SDS in $50 \mathrm{mM}$ Tris, $150 \mathrm{mM} \mathrm{NaCl}, \mathrm{pH}$ 7.6; TBS) containing nucleases (Thermo Fisher Scientific) and protease and phosphatase inhibitor cocktail (Thermo Fisher Scientific). After sonication with an ultrasonic homogenizer (Biologics, Inc.) and centrifugation at $13,000 \mathrm{~g}$ for $30 \mathrm{~min}$ at RT using a $5415 \mathrm{R}$ centrifuge (Eppendorf), the resulting supernatant was collected, and the insoluble pellet was resuspended in $70 \%$ formic acid. Formic acid was evaporated from the samples in a vacuum concentrator (Concentrator plus 5305, Eppendorf), and samples were resuspended in NuPAGE LDS Sample Buffer (Invitrogen). For the soluble fraction, protein concentrations were estimated using the Pierce BCA Protein Assay (Thermo Fisher Scientific). Samples were incubated with NuPAGE Sample Reducing Agent (Invitrogen) and NuPAGE LDS Sample Buffer (Invitrogen) at $75^{\circ} \mathrm{C}$ for $10 \mathrm{~min}$. Equal amounts of protein (human brain tissue: $20 \mu \mathrm{g}$; necroptosis-positive SH-SY5Y cells: $10 \mu \mathrm{g}$ ) were loaded onto NuPAGE 4-12\% Bis-Tris Protein Gels (Life Technologies) in MOPS-SDS running buffer (Alfa Aesar), electrophoresed at $150 \mathrm{~V}$ for $60 \mathrm{~min}$, and then transferred to Amersham Protran Supported 0.2 NC membranes (GE Healthcare). After blocking either with $5 \%$ non-fat dried milk (AppliChem) in PBS $0.1 \%$ Tween20 (PBST) or with 5\% BSA (Sigma-Aldrich) in TBS 0.1\% Tween-20 (TBST) for $1 \mathrm{~h}$ at RT, the membranes were reacted with primary antibodies (Suppl. Table 1) in PBST or TBST overnight at $4^{\circ} \mathrm{C}$ and detected with species-specific secondary antibodies conjugated to horseradish peroxidase (HRP) (Dako). Bands were visualized using either SuperSignal West Pico or Dura chemiluminescent substrate (Thermo Fisher Scientific) and imaged with an Amersham Imager 600 (GE Healthcare). All blots were stripped of the bound antibodies and reprobed with GAPDH to verify equal protein loading. Intensity of protein bands was quantified with ImageJ software and normalized to that of GAPDH. All values are reported as ratio of band density of necroptotic protein to band density of GAPDH. For the formic acid fraction, $15 \mu \mathrm{l}$ was loaded per sample after the addition of NuPAGE Sample Reducing Agent. All remaining procedures were as described above.

\section{Statistical analysis}

Statistical analyses were performed using SPSS and Graphpad Prism software. A one-way ANOVA or a non-parametric one-way ANOVA (Kruskal-Wallis test), followed by Tukey's or 
Dunn's post-hoc test for multiple comparisons, was used to determine the significance of differences across the three groups. To control for age- and sex-associated changes, age at death and sex were included in all regression analyses. Comparisons between two antibodies were performed using Wilcoxon test (pMLKL and pTDP-43) or Sign test (pMLKL and CK1ס) for matched pairs. To examine correlations between GVD and other AD-defining parameters, partial correlation analysis adjusted for age, sex and PMI was applied. To estimate the effect of explanatory variables on $A D$ progression, we conducted a linear regression analysis. Data are presented as mean \pm SEM. Statistical significance was accepted at $p<0.05$ level. ${ }^{*} p<$ $0.05 ;{ }^{* *} p<0.01 ;{ }^{* * *} p<0.001 ;{ }^{* * * *} p<0.0001$.

\section{Results}

\section{Activated necrosome components are localized in GVD lesions in the AD brain}

To investigate the presence and morphological features of necrosome components in degenerating neurons in $A D$, we carried out pathological analyses on human post-mortem clinical $A D(n=23)$, p-preAD $(n=24)$ and non-AD control $(n=16)$ brain tissues. Immunohistochemical analysis showed positive immunoreactivity of pRIPK1 (Figure 1a, d), pRIPK3 (Figure 1b, e) and pMLKL (Figure 1c, f) in all 23 AD cases, 22 out of 24 p-preAD cases and two out of 16 non-AD control cases. Specifically, pRIPK1, pRIPK3 and pMLKL were located within the granular component of GVD or at the membranes of the vacuoles, but not inside the lumen of the vacuoles (Figure 1d-f). Anatomically, these proteins were detected outside the nucleus in the cytoplasmic compartment of neurons in the hippocampal subfields $\mathrm{CA} 1, \mathrm{CA} 2 / 3, \mathrm{CA} 4$, dentate gyrus, subiculum, entorhinal cortex, temporal cortex (Brodmann area 36), hypothalamus, amygdala, and frontal cortex, exhibiting the pattern of GVD. Interestingly, all the phosphorylated proteins were detected in the GVDn+ (GVDn+, i.e. GVD with activated necrosome) neurons but not in neighboring neurons without GVD granules (Figure 1a-c), astrocytes or microglial cells (Figure 2). Antibody specificity was confirmed by the use of respective blocking peptides as well as by dephosphorylation, which eliminated the phosphorylated targets of anti-pRIPK1, anti-pRIPK3, and anti-pMLKL. After dephosphorylation, antibodies against non-phosphorylated RIPK1 and RIPK3 stained GVD lesions, which were negative for the anti-RIPK3 staining before dephosphorylation (Suppl. Figures 1 and 2).

To determine whether the activated necroptotic markers were indeed localized in GVD inclusions, we performed double-immunofluorescence analysis of the activated necroptotic marker pMLKL with the established GVD markers, i.e. anti-CK1ס (Figure 3i-I) and anti-pTDP43 (Figure 3m-p). We observed that all three antibodies consistently yielded a similar proportion of GVD-positive neurons, with slight variation in immunoreactivity in particular GVD 
granules inside cell bodies of neurons (Figures 3a-h and 6, Suppl. Figure 5). Double immunolabeling of anti-pMLKL with anti-pRIPK1 or anti-pRIPK3 indicated that all neurons show colocalization of pMLKL with pRIPK1 and pRIPK3 in AD and p-preAD brain tissues. To further confirm these results, we validated the presence of pMLKL in GVD inclusions by using a second antibody against pMLKL, which showed an identical staining pattern (Suppl. Figure $3 b, d)$. As pMLKL is considered to be a principal component of the necrosome complex that finally executes necroptosis, we used the anti-pMLKL antibody for further analysis of necroptotic cell death.

To assess the accuracy of GVD-staining by anti-pMLKL, a semi-quantitative comparison of pMLKL-positive GVD-containing neurons with pTDP-43-positive GVD-containing neurons was performed in a subset of 31 representative cases. This analysis showed similar semiquantitative scores for GVD pattern stained with anti-pMLKL as well as with anti-pTDP-43 in the medial temporal lobe (MTL) regions of 12 AD (Suppl. Figure 4a), nine p-preAD (Suppl. Figure $4 b$ ) and ten non-AD cases. In the six MTL regions of $A D$ cases, similar amounts of neurons were marked by anti-pMLKL and anti-pTDP-43. However, in the p-preAD group, more GVD-positive neurons were identified in the entorhinal cortex with anti-pMLKL than with antipTDP-43 ( $p=0.045$; Wilcoxon matched-pairs signed rank test). We found differences in the pMLKL expression in GVD as measured by the GVD severity score of MTL among AD, $p$ preAD and non-AD control cases (Suppl. Figure 4c), as well as in the GVD severity score of MTL detected with anti-pTDP-43 (Suppl. Figure 4d). Moreover, a separate quantitative analysis of the percentage of GVD-positive neurons revealed no differences between antipMLKL and anti-CK1ס (hippocampal CA1: $p=0.253$; frontal cortex layer III: $p=0.077$; Sign test).

The distribution pattern of pMLKL-positive GVD lesions in the brain fits with the previously

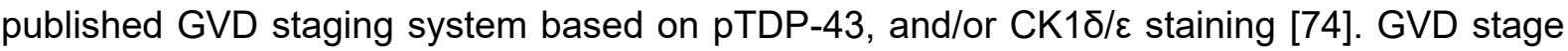
gradually increased from non-AD controls to p-preAD, reaching the highest stages in $A D$ cases, indicating a progressive expansion of pMLKL-immunoreactive GVD lesions from the hippocampus to the frontal cortex (Figure 4), and proving its capability to mark GVD even in the latest stage as good as pTDP-43. Using the anti-pMLKL antibody, we were able to detect GVD-positive cells in the CA1 region of two control cases without PART, which were negative for anti-pTDP-43. Finally, when controlled for age, sex and PMI, we obtained significant positive partial correlations between the PMLKL-GVD stage and the ABMTL phase, Braak NFT stage, CERAD score, CDR score, NIA-AA degree of AD pathology, and the clinical progression from control to p-preAD and AD (see Suppl. Table 2a, b for $r$ - and $p$-values), from which the association with the Braak NFT stage was the strongest. 


\section{GVD-related necrosome activation is associated with Tau but not $A \beta$ pathology}

We histopathologically evaluated the presence of intracellular pTau and extracellular $A \beta$ aggregates together with necrosome components in the hippocampal formation of AD cases. We did not find colocalization between $A \beta$ and pRIPK1, pRIPK3 or pMLKL. However, we observed co-expression of pMLKL and pTau in GVD-bearing neurons in AD and p-preAD cases (Figure 5f-o). Specifically, we observed three phenotypes of AD-affected neurons, in which the presence of pMLKL was indicative of GVD: 1) pMLKL-positive and pTau-negative neurons (Figure 5o, arrowhead); 2) pMLKL- and pTau-positive neurons (Figure 5o, arrow); 3) pTau-positive tangles and pre-tangles in the absence of pMLKL-immunoreactive GVD (Figure $5 \mathrm{j}$, arrow). These findings were confirmed in $1 \mathrm{~mm}$ thick temporal cortex sections of an $A D$ case processed by the CUBIC method (Table 1; case 3). Here, we analyzed the topographical relationship of the lesions in pyramidal neurons in their 3-dimensional orientation and confirmed all three different types of neurons (Suppl. Movie 1), as previously identified on 5 $\mu \mathrm{m}$ sections. Finally, to determine the effect of explanatory variables on AD progression, we performed a linear regression analysis. We obtained a significant positive regression for the Braak NFT stage $(p<0.001)$ and for the GVD stage $(p=0.045)$; however, four other factors, including the ABMTL phase, age, sex, or PMI did not have an effect on AD progression in this model (Suppl. Table 3a, b).

pMLKL expression in GVD is associated with lower neuronal density in the CA1 region of the hippocampus and in layer III of the frontal cortex

To evaluate a potential link between the presence of GVDn+ neurons (exhibiting necrosome components such as pMLKL) and neuronal loss, we assessed the number of GVDn+ neurons in relation to the total number of neurons in the CA1 region of the hippocampus and in layer III of the frontal cortex (Figure 6a, b). Using the Kruskal-Wallis test followed by Dunn's post-hoc analysis, we observed higher percentages of GVDn+ neurons in CA1 of the AD group (41.8 \pm $2.2 \%)$ compared to $p$-preAD $(9.7 \pm 1.9 \%, p<0.0001)$ and control cases $(0.1 \pm 0.1 \%, p<$ 0.0001$,$) . In addition, the p-preAD group showed an increased occurrence of GVD-bearing$ neurons in relation to the control group $(p=0.0052)$. In layer III of the frontal cortex, we found a less prominent GVD pattern, which was only present in the AD cohort $(1.9 \pm 0.5 \%)$, and which significantly differed from the other two groups ( $p<0.0001$, Kruskal-Wallis). Additionally, when we controlled for age, sex or PMI, linear regression analyses showed a significant effect of the independent variable GVDn+ neurons on a dependent variable, i.e. AD progression, in both CA1 $(\beta=0.034, p<0.001)$ and frontal cortex layer III $(\beta=0.242, p<0.001)$. Furthermore, we analyzed the neuronal density in these two brain regions by one-way ANOVA followed by Tukey's post-hoc analysis (Figure $6 \mathrm{c}, \mathrm{d}$ ) and found a decrease in the hippocampal neuronal 
density in AD $\left(80.9 \pm 5.5\right.$ cells $\left./ \mathrm{mm}^{2}\right)$ compared to $p$-preAD $\left(108.5 \pm 3.1 \mathrm{cells} / \mathrm{mm}^{2}, p=0.0002\right)$ and to the control group (135.2 $\left.\pm 5.6 \mathrm{cells} / \mathrm{mm}^{2}, p<0.0001\right)$. A lower neuronal density was also detected in $p$-preAD compared to control hippocampus $(p=0.001)$. In layer III of the frontal cortex, $A D$ cases showed a neuronal density reduction $\left(98.9 \pm 1.8\right.$ cells $\left./ \mathrm{mm}^{2}\right)$ in comparison to p-preAD $\left(111.1 \pm 1.5 \mathrm{cells} / \mathrm{mm}^{2}, p=0.0001\right)$ and to the control group (113.2 \pm 2.4 cells $\left./ \mathrm{mm}^{2}, p<0.0001\right)$. These results were confirmed when we controlled for age. The effect of neuronal density on AD progression was significant in a linear regression analysis for both CA1 $(\beta=-0.016, p<0.001)$ and frontal cortex layer III $(\beta=-0.039, p<0.001)$. Partial correlation analyses controlled for age, sex and PMI showed a negative relationship between the percentage of pMLKL-positive GVD-bearing neurons and cell density in CA1 region of the hippocampus ( $r=-0.643, p<0.001$; Suppl. Table $4 a, b)$ and in layer III of the frontal cortex ( $r$ $=-0.347, p=0.008$; Suppl. Table 5a, b). The decreasing neuron density in the CA1 region and layer III of the frontal cortex showed similar associations with the Braak NFT stage, ABMTL phase, CERAD score for neuritic plaques, NIA-AA degree of AD pathology and the pMLKLbased GVD stage (Suppl. Tables $4 a$, b and 5a, b). Finally, to clarify whether GVDn+ neurons associate in a similar way with neuronal loss as GVD neurons labeled with a standard marker, we carried out the same quantitative analysis using an established GVD marker, CK1ס. We found no statistical differences between the percentage of positive GVD neurons obtained with anti-pMLKL and anti-CK1ס (two-tailed Sign-test; CA1: $p=0.253$; layer III: $p=0.077$ ), showing that both antibodies similarly detected GVD granules. Regardless of the marker, we detected the same differences between groups in the percentage of positive GVD neurons and in the neuronal density (Suppl. Figure 5). Furthermore, we obtained partial correlations between neuronal loss and CK1ס-positive GVD neurons (CA1: $r=-0.667, p<0.001$; layer III: $r=-0.255$, $p=0.055)$, which did not substantially differ from those observed with GVDn+ neurons (CA1: $r=-0.643, p<0.001$; layer III: $r=-0.347, p=0.008)$.

\section{Histopathological characterization of non-phosphorylated necroptosis-related proteins}

Since AD-associated necroptosis was previously studied mainly using non-phosphorylated RIPK1, RIPK3 and MLKL, we further investigated the expression of non-phosphorylated proteins, which allowed us to determine their expression pattern and cellular location under physiological conditions and in AD. Immunoreactive RIPK1 protein was present in both neuronal and non-neuronal cells (Figures 7a-e and 8a). Specifically, we detected RIPK1positive neurons with a tangle-like staining pattern in 11 out of $12 \mathrm{AD}$ cases, two out of nine $p$ preAD cases, and in none of the non-AD cases (Figure 7c). GVD granules were RIPK1-positive in all $12 A D$ cases, six out of nine p-preAD and in none of ten non-AD cases (Figure 7a-d). We found a substantial increase in RIPK1 levels in astrocytes, which resembled the morphology of reactive astrocytes, in all $12 A D$ and nine p-preAD cases (Figures $7 a-c$ and $8 a$ ). In non-AD 
control cases, neurons were not positive and activated astrocytes showed RIPK1 expression in six out of ten cases (Figure 7e). Examination of sections stained with an anti-RIPK3 antibody revealed a positive immunoreaction in the neuronal cytoplasm in 11 out of $12 \mathrm{AD}$, all nine $\mathrm{p}$ preAD, and in six out of ten non-AD control cases (Figures $7 \mathrm{f}-\mathrm{i}$ and $8 \mathrm{~b}$ ), and in dystrophic neurites of neuritic plaques in ten out of $12 A D$ and in three out of nine p-preAD cases (Figure $7 f)$. No neuritic plaques were observed in the non-AD control cases. Astrocytes and microglial cells did not exhibit RIPK3 (Figure 8b). Interestingly, although RIPK3-positive cytoplasmic granules were observed in AD, p-preAD and even non-AD cases (Figures 7f, g and 8b), GVD lesions visualized by hematoxylin counterstaining remained negative for non-phosphorylated RIPK3 (Figure 7h). Only after dephosphorylation treatment, we detected RIPK3immunoreactive GVD granules (Suppl. Figure 2), proving the presence of the phosphorylated RIPK3 protein in GVD lesions. We did not immunohistochemically characterize the MLKL protein as none of the tested antibodies worked properly on the human paraffin-embedded tissue available in our laboratory. However, the anti-MLKL antibody worked in western blots, which showed increased levels of MLKL in p-preAD and symptomatic AD cases (Figure 9a, d).

\section{pMLKL-immunoreactive astrogliosis in the CA4 and CA3 region of the hippocampus is not associated with $A D$}

In addition to the detection of neuronal GVD inclusions in p-preAD and AD cases, we observed pMLKL-positive astrocytes restricted to the CA4 and the adjacent CA3 region of the hippocampus (Suppl. Figure 6). Out of 63 examined cases, 18 cases (i.e. six out of 23 AD cases, ten out of 24 p-preAD cases, and two out of 16 non-AD control cases) exhibited pMLKLimmunoreactive hippocampal astrocytes. These pMLKL-positive astrocytes were not detected using anti-pRIPK1 and anti-pRIPK3. To examine a possible effect of relevant variables, including the pMLKL-GVD stage, the Braak NFT stage, the ABMTL phase and the AD progression, on pMLKL-immunoreactive astrogliosis, we carried out separate linear regression analyses. None of the independent variables, including age, sex and PMI, had a significant effect on pMLKL-positive astrocytes (Suppl. Table 6).

\section{Biochemical analysis of necroptosis-related proteins}

Finally, we determined the levels of three proteins involved in the necroptosis pathway, i.e. RIPK1, pRIPK1, and MLKL, using western blotting (Figure 9a, Suppl. Figure 7). We detected no changes in RIPK1 levels while comparing three cohorts (Figure 9b). However, we observed an increase in pRIPK1 expression in AD brains compared to the non-AD group $(p=0.0068)$ (Figure 9c). Furthermore, we detected higher MLKL levels in AD $(p<0.0001)$ and $p$-preAD $(p$ $=0.0017$ ) tissues in relation to the non-AD control group (Figure 9d). These results from a 
biochemical analysis of the levels of RIPK1, pRIPK1 and MLKL are in line with the previously described immunohistochemical findings. SH-SY5Y neuroblastoma cells were stimulated for necroptosis and used as a positive control for the changes observed in necroptosis-related proteins in human tissue. Western blots indicated that most antibodies were able to detect the respective proteins in necroptotic SH-SY5Y neuroblastoma cells, but did not detect RIPK3, pRIPK3 and pMLKL in significant amounts in human brain homogenates from AD, p-preAD, and control non-AD cases (Suppl. Figure 7).

\section{Discussion}

In this study, we provide evidence for the presence of the three activated components of the necrosome machinery, i.e. pRIPK1, pRIPK3, and pMLKL, in degenerating neurons in AD and preclinical stages of $A D$ pathology. Phosphorylation-mediated activation of necrosome proteins is crucial for necrosome assembly and subsequent necroptosis execution [11]. Here, the activated necrosome components were observed in GVD lesions (referred to as GVDn+ if the necroptosis executor pMLKL was detected), and correlated with neuronal loss in ADaffected brain regions, such as the hippocampal CA1 region and the frontal cortex layer III. Further validation using established GVD markers (pTDP-43 and CK1ס) confirmed the presence of the activated necrosome in GVD, and the quantification of GVD-positive neurons using anti-CK1 $\delta$ and anti-pMLKL showed no differences between the two markers. To the best of our knowledge, this is the first work that demonstrates the presence of activated necrosome proteins in the $A D$ and p-preAD brain, and their relationship to GVD pathology and neuronal loss. With these findings, we provide new insights on the involvement of necroptosis in $A D$ [10], by specifying the neuropathological lesions in which the necrosome complex is located, their anatomical distribution pattern, and their relation to changes in neuronal densities in affected brain regions.

Some reports indicate the presence of potentially toxic proteins in GVD granules, including proteins related to AD pathology [35], such as pTau [15] and phosphorylated A $\beta$ (Ser26) [37]. Nevertheless, so far none of the necroptotic proteins were linked to GVD. Although some reports suggested the presence of cleaved caspase-3 in GVD lesions, the absence of apoptotic morphological features in the cells argues in favor of the hypothesis that GVD is not associated with apoptosis [35, 63, 65, 67]. In contrast to caspase-dependent apoptosis, execution of pMLKL-dependent necroptosis involves oligomerization of pMLKL, together with a disruption of the plasma membrane [11]. The accumulation of pMLKL in GVDn+ may also be toxic to cells, probably altering membranes of GVD granules in a similar way to the necroptosis-related changes in the plasma membrane. Arguments in favor of this hypothesis are our findings that the necrosome components were generally present in GVD lesions in AD 
and p-preAD cases, specifically detected in the granules and in the membrane of the GVD vacuoles, and showed an inverse association with neuronal density in regions exhibiting GVD. Using pMLKL immunostaining, we categorized anatomical brain regions that were gradually affected by GVD as AD pathology progresses, which was in line with the previously published stages of GVD expansion in the brain [74]. In accordance with earlier reports, we also detected GVDn+ neurons in few control cases (two out of 16), but far less advanced (stage I) compared to $A D$ cases $[3,74,75,81]$. The pMLKL-based GVD stages highly correlated with the ADdefining parameters, including the ABMTL phase, Braak NFT stage, and the CERAD score, of which the association with the Braak NFT stage representing Tau pathology, was the strongest one.

Previous studies suggested that GVD lesions are involved in the degeneration of neuronal cells in $A D$, and strongly associated with $A D$-related specific insults, such as $p T a u$ and $A \beta$ pathology [74]. However, the relationship between $p T a u, A \beta$ and GVD is still a matter of debate and the underlying mechanisms remain unknown. Some reports showed the occurrence of both pTau and GVD pathology in the same neuron [32, 39, 80], while others contradicted this $[43,49]$. Here, we observed the existence of pTau and GVDn+ neurons, as well as of neurons with either $\mathrm{pTau}$ or GVDn+ lesions alone, which points to a vague relationship between these two lesions in AD pathogenesis. Regarding the neuronal density, we observed a mild decrease in neuronal density in the frontal cortex layer III only in AD cases (compared to control cases). However, in the CA1 region, a significant decrease was already observed in p-preAD cases, with symptomatic $A D$ cases showing the highest loss of neurons. These findings are in line with previously published results $[70,79]$. The correlation between the increased percentage of GVDn+ neurons and the decreased neuronal density in the affected brain regions, suggests a possible role for GVD in neuronal loss in AD. Since $A \beta$ pathology is present in neocortical areas before the hippocampus gets affected [72], we would expect $A \beta$-associated neuronal loss to be more advanced in the frontal cortex than in the hippocampus. Therefore, it is unlikely that the observed reduction in neuronal density, which is most pronounced in hippocampal areas, is related to amyloid burden. This leaves GVD and NFT pathology as potential drivers of neuronal demise in $A D$, which is supported by a recent report [19]. The strong correlations of the Braak NFT stage and the pMLKL-GVD stage with neuronal loss in both the CA1 area and the frontal cortex layer III further support this interpretation. Since the GVD stage was not found to be associated with other tauopathies, such as progressive supranuclear palsy or argyrophilic grain disease [74], we speculate that (GVDn+)-associated neuronal death is specific to AD. Furthermore, GVD lesions have been reported in Tau transgenic mice (24 months) and in double transgenic mice harboring both human APP and Tau transgenes [36, 40], but not in amyloid mouse models. These data further support the hypothesis that 
pathological Tau might play a role in GVD-associated cell death. In line with this, we found that the GVD stage and the Braak NFT stage, but not the ABMTL phase, were able to predict AD progression from non-AD to $p-p r e A D$ and $A D$ in a linear regression model. Previous studies already showed that $A \beta$ deposition, although it is a defining feature for $A D$, correlates less with cognition compared to NFTs, synaptic dysfunction or neuronal loss, which strongly predict memory decline $[19,20]$. Given the above, instead of pointing to a causal relationship between pTau and GVD, we rather speculate that the combined and accumulative effect of toxic proteins detected in GVD (e.g. pTau, among many others [35]) is responsible for changes in neuronal homeostasis, resulting in (GVDn+)-associated neuronal death in AD. Interestingly, recent findings showed that GVD is associated with C9orf72 mutation carriers in frontotemporal lobar degeneration (FTLD) and ALS, which supports the hypothesis that the accumulation of toxic proteins, i.e. dipeptide repeat proteins (DPR) in C9orf72 mutation carriers, could stimulate the formation of GVD in affected neurons [57]. Finally, it is of importance to mention that the possible contribution of GVDn+ to neuronal loss in AD does not exclude the involvement of other neuronal death mechanisms, including apoptosis [65] or NFTinduced neuron degeneration leading to ghost tangles $[5,7,60]$.

Cell death can result from passive or accidental signals when initiated by a harsh physical trigger, or it can occur via controlled cell signaling programs as a part of development, homeostasis, or pathological processes [42]. Since the CA1 region of the hippocampus is most vulnerable to $A D$-associated degeneration [82], hippocampal atrophy rates are commonly used in both diagnosing and monitoring the progression of $A D$, which typically start 10 years before the onset of clinical signs of dementia [6]. In general, regulated forms of cell death manifest through a sequence of events involving a trigger, initiator, mediator, and an executor [76]. Once initiated, cell death execution occurs within approximately 24 hours. We found all three activated necrosome components of the necroptosis pathway, i.e. pRIPK1 (initiator), pRIPK3 (mediator) and pMLKL (executor), present in GVD-bearing neurons in symptomatic $A D$ and p-preAD brains. Activation of necroptosis has been shown to be implicated in $A D$ neurodegeneration [10]. Nevertheless, that study focused on the characterization of nonphosphorylated native proteins of necroptosis, i.e., RIPK1, RIPK3 and MLKL, with the exception of pMLKL, in the temporal cortex of $A D$ and control cases. The native forms of RIPK1 and RIPK3 seem to have pleiotropic functions, such as roles in inflammation, controlling the disease-associated state of microglia in $A D$, and energy homeostasis [51, 78, 83]. Here, we observed RIPK1 immunoreactivity in non-AD, p-preAD, and $A D$ astroglial cells and in p-preAD and AD neuronal cells (GVD and tangle-like staining pattern). RIPK3 was physiologically found in the cell bodies of neurons of non-AD, p-preAD and AD cases, and in neuritic plaques of $p-$ preAD and $A D$ cases. While non-phosphorylated RIPK3 was not detected in GVD lesions, after 
the dephosphorylation treatment we observed the signal of anti-RIPK3, confirming the presence of the phosphorylated RIPK3 protein in GVD granules. Unfortunately, we were unable to find an antibody for non-phosphorylated MLKL that worked in paraffin embedded tissue. Given the presence of non-phosphorylated necroptosis markers in non-AD cases, the phosphorylation status of all three necrosome components and the necrosome assembly must be considered when determining necroptosis activation in $A D$. In contrast to the systematically detected colocalization between the components of the activated necrosome complex (pRIPK1, pRIPK3, pMLKL), we never found colocalization between non-phosphorylated RIPK1 and RIPK3, which differs from previously reported findings [10]. Moreover, contrarily to what has been reported about pMLKL localization, we detected PMLKL in GVD lesions in neuronal cells, but not in oligodendrocytes, and microglia [10]. Astroglial pMLKL-expression was seen in the CA4 and CA3 sector of the hippocampal formation in similar levels in a subset of non-AD, p-preAD and $A D$ cases. No association between pMLKL-positive astrocytes and pMLKL-GVD stage, AD progression or other AD-relevant variables (ABMTL and Braak NFT stage) was observed in regression models, arguing against a potential role of these astrocytes in AD pathogenesis. Furthermore, pRIPK1 and pRIPK3 were not present in pMLKL-positive astrocytes, which excludes the recruitment of a functional necrosome in these cells.

The exclusive presence of the three phosphorylated necrosome components in GVD points to a specialized sequestration mechanism in neuronal cells, which is strongly associated with $A D$ and its related neuropathological parameters. In turn, this supports the hypothesis that GVD is the morphological correlate of necrosome-activation in $A D$, presumably involved in necroptotic neuronal loss. However, if all neurons exhibiting GVDn+ were in the status of ongoing necroptosis, neuronal death would be executed within a few weeks rather than within approximately 50 years in AD [52]. Furthermore, an approximately 20-year lifetime of NFTaffected neurons [46] is another counterargument to an immediate execution of necroptotic cell death in AD. We hypothesize that different developmental steps of GVDn+ formation exist within a neuron, from mild to severe, reflecting a progressive deterioration of the pyramidal neurons, which eventually are destined to die. The execution of necroptotic cell death requires the activation of MLKL by phosphorylation, which directly facilitates cell death. Earlier studies indicated that phosphorylation of MLKL residues induces conformational changes in the protein. The resulting four-helical bundle $(4 \mathrm{HB})$ domain exposure and oligomerization through the $\mathrm{N}$-terminal domain lead to pMLKL translocation and plasma membrane rupture, eventually causing cell death $[25,47]$. Here, we found pMLKL clustered in the membrane and the granular component of GVD vacuoles, rather than present in the plasma membrane, disabling pMLKL to execute necroptotic cell death. This possibly protective mechanism of delayed necroptosis might become ineffective as cell dysfunction increases, eventually reaching the level where 
GVD granules might release the trapped activated necroptotic complex, leading to cellular suicide in $A D$.

The activation of necroptosis has also been reported in other neurodegenerative disorders, including ALS [31, 55], and in multiple sclerosis [50, 59]. In multiple sclerosis, cell damage specifically occurs in oligodendrocytes. Increased levels of tumor necrosis factor $\alpha$ (TNF $\alpha$ ), a prominent pro-inflammatory cytokine, have been shown to bind to TNFR1 complex, and to trigger necroptosis in oligodendrocytes. In ALS, patient-derived astrocytes induced necroptosis exclusively in motor neurons [55]. Furthermore, optineurin, a component of the TNFR1 signaling pathway that directly regulates RIPK1 protein turnover, has been implicated in axonal degeneration and motor neuron dysfunction in ALS [31]. A recent study has shown a link between GVD and the C9orf72 hexanucleotide repeat mutation in ALS and FTLD patients [57]. However, since necroptosis-related proteins were not targeted in this study, it remains open whether the same GVD/necrosome-related lesions are associated with neuronal loss in ALS and FTLD. In future research, the phosphorylation status of necroptotic proteins should be reconsidered when analyzing necroptosis in the above-mentioned pathological conditions. Focusing on native proteins, i.e. RIPK1, RIPK3, and MLKL, we detected these proteins not only in AD but also in non-AD control cases. This may be indicative of physiological levels and not necessarily of pathological conditions or execution of necroptosis, for which post-translational modifications and activation of necrosome components are required.

One of the limitations of this study is a relatively small sample size of 63 cases, which made it difficult to statistically analyze linear model terms with more than three variables. Therefore, the scores of a linear regression analysis using GVD, ABMTL phase and Braak NFT stage as independent variables must be interpreted with caution. Although the western blot analysis of cells, in which necroptosis was induced, confirmed specificity of the antibodies against the necrosome components, we were not able to detect RIPK3, pRIPK3 and pMLKL in brain homogenates by western blotting. This might be due to too low concentrations of the respective proteins in the brain lysates and lack of antibodies suitable to detect these proteins in western blot. Moreover, necrosome complex formation, oligomerization, and cleavage products may explain the difficulties in analyzing pRIPK3 and pMLKL in western blot [41]. These aspects need further clarification.

In conclusion, our findings indicate the presence of the activated necrosome complex in GVD granules, and its association with neuronal loss in areas that are most affected by $A D$ neurodegeneration, as well as in less affected regions. Given the lifetime of degenerating neurons in $A D$, which is longer than the $24 \mathrm{~h}$ required for regular necroptosis [46], and the GVD-specific localization of the necrosome complex in AD-susceptible brain regions, we hypothesize that the observed lesions represent a delayed type of necroptosis. This 
necroptosis-related form of a long-term neurodegenerative process involving GVD appears to be of major relevance for AD-associated neuronal loss. As until now no disease-modifying treatment against $A D$ has been clinically established, neuronal death mechanisms could be an interesting novel target for the treatment of $A D$. Therapeutic targeting of cell death mechanisms may be a more effective strategy to stop $A D$ progression, compared to for example $A \beta$-directed therapies, as the disease would be targeted at more advanced stages preventing neuronal degeneration [72]. While further studies are needed to pinpoint the nature of the connection between the necrosome components and GVD, the findings reported here provide new insights into AD-related neuronal death, especially highlighting the potential importance of the GVD-associated necrosome complex.

\section{Acknowledgements}

The authors gratefully acknowledge the assistance of Mrs. Alicja Ronisz. They also thank Mathias De Decker (Laboratory for Neurobiology, VIB-KU Leuven, Belgium) for providing SHSY5Y cells. Also, we thank the VIB Imaging Core Facility in Leuven for expert assistance and overall technical support in super-resolution imaging of cleared tissue using spinning disk confocal microscopy. The study was supported by: Fonds Wetenschappelijk Onderzoek (FWO) G0F8516N (DRT, RV); C1-internal funds from KU Leuven C14-17-107 (DRT); Vlaamse Impulsfinanciering voor Netwerken voor Dementie-onderzoek (IWT 135043) (RV, BDS, DRT) and a Methusalem grant of the Flemish Government and the KU Leuven to BDS.

\section{Online Content}

Supplementary figures, along with additional Extended Data display items and SourceData, are available in the online version of the paper. References unique to these sections appear only in the online paper.

\section{Competing interests statement}

DRT received consultant honorary from GE-Healthcare (UK) and Covance Laboratories (UK), speaker honorary from Novartis Pharma AG (Switzerland), travel reimbursement from GEHealthcare (UK) and UCB (Belgium) and collaborated with Novartis Pharma AG (Switzerland), Probiodrug (Germany), GE-Healthcare (UK), and Janssen Pharmaceutical Companies (Belgium). BDS collaborated with Janssen Pharmaceutical companies (Belgium), Abbvie (USA) and received consulting fees from Eisai (Japan). None related to the work in this paper. RV's institution has clinical trial agreements (RV as PI) with AbbVie, Genentech, Novartis, and Roche, material transfer agreements (RV as PI) with Avid a subsidiary of EliLilly, and consultancy agreements (RV as PI) with Prevail Therapeutics and Rodin Therapeutics. CAFvA received honoraria from serving on the scientific advisory board of Nutricia $\mathrm{GmbH}$, Roche, Dr. 
Willmar Schwabe GmbH and Honkong University Research council and has received funding for travel and speaker honoraria from Nutricia $\mathrm{GmbH}$, Lilly Deutschland $\mathrm{GmbH}$, Desitin Arzneimittel GmbH, Biogen, Roche and Dr. Willmar Schwabe GmbH \&Co. KG.

\section{References}

1. Association A (2017) 2017 Alzheimer's disease facts and figures. Alzheimer's Dement 13:325-373. doi: 10.1016/j.jalz.2017.02.001

2. Ball MJ (1977) Neuronal loss, neurofibrillary tangles and granulovacuolar degeneration in the hippocampus with ageing and dementia. Acta Neuropathol 37:111-118. doi: 10.1007/BF00692056

3. Ball MJ (1978) Topographic distribution of neurofibrillary tangles and granulovacuolar degeneration in hippocampal cortex of aging and demented patients. A quantitative study. Acta Neuropathol 42:73-80. doi: 10.1007/BF00690970

4. Balusu S, Brkic M, Libert C, Vandenbroucke RE (2016) The choroid plexuscerebrospinal fluid interface in Alzheimer's disease: more than just a barrier. Neural Regen Res 11:534. doi: 10.4103/1673-5374.180372

5. Bancher C, Brunner C, Lassmann H, Budka H, Jellinger K, Wiche G, Seitelberger F, Grundke-lqbal I, lqbal K, Wisniewski HM (1989) Accumulation of abnormally phosphorylated $\mathrm{T}$ precedes the formation of neurofibrillary tangles in Alzheimer's disease. Brain Res 477:90-99. doi: 10.1016/0006-8993(89)91396-6

6. Barnes J, Bartlett JW, van de Pol LA, Loy CT, Scahill RI, Frost C, Thompson P, Fox NC (2009) A meta-analysis of hippocampal atrophy rates in Alzheimer's disease. Neurobiol Aging 30:1711-1723. doi: 10.1016/j.neurobiolaging.2008.01.010

7. Braak F, Braak H, Mandelkow E-M (1994) A sequence of cytoskeleton changes related to the formation of neurofibrillary tangles and neuropil threads. Acta Neuropathol 87:554-567. doi: 10.1007/BF00293315

8. Braak H, Alafuzoff I, Arzberger T, Kretzschmar H, Del Tredici K (2006) Staging of Alzheimer disease-associated neurofibrillary pathology using paraffin sections and immunocytochemistry. Acta Neuropathol 112:389-404. doi: 10.1007/s00401-006-0127z

9. Braak H, Braak E (1991) Neuropathological stageing of Alzheimer-related changes. Acta Neuropathol 82:239-259. doi: 10.1007/BF00308809

10. Caccamo A, Branca C, Piras IS, Ferreira E, Huentelman MJ, Liang WS, Readhead B, Dudley JT, Spangenberg EE, Green KN, Belfiore R, Winslow W, Oddo S (2017) Necroptosis activation in Alzheimer's disease. Nat Neurosci 20:1236-1246. doi: $10.1038 / \mathrm{nn} .4608$ 
11. Cai Z, Jitkaew S, Zhao J, Chiang HC, Choksi S, Liu J, Ward Y, Wu LG, Liu ZG (2014) Plasma membrane translocation of trimerized MLKL protein is required for TNF-induced necroptosis. Nat Cell Biol 16:55-65. doi: 10.1038/ncb2883

12. Chen X, Li W, Ren J, Huang D, He W, Song Y, Yang C, Li W, Zheng X, Chen P, Han J (2013) Translocation of mixed lineage kinase domain-like protein to plasma membrane leads to necrotic cell death. Cell Res 24:105. doi: 10.1038/cr.2013.171

13. Cho Y, Challa S, Moquin D, Genga R, Ray TD, Guildford M, Chan FK-M (2009) Phosphorylation-driven assembly of the RIP1-RIP3 complex regulates programmed necrosis and virus-induced inflammation. Cell 137:1112-1123. doi: 10.1016/j.cell.2009.05.037

14. Crary JF, Trojanowski JQ, Schneider JA, Abisambra JF, Abner EL, Alafuzoff I, Arnold SE, Attems J, Beach TG, Bigio EH (2014) Primary age-related tauopathy (PART): a common pathology associated with human aging. Acta Neuropathol 128:755-766. doi: 10.1007/s00401-014-1349-0

15. Dickson DW, Ksiezak-Reding H, Davies P, Yen S-H (1987) A monoclonal antibody that recognizes a phosphorylated epitope in Alzheimer neurofibrillary tangles, neurofilaments and tau proteins immunostains granulovacuolar degeneration. Acta Neuropathol 73:254-258. doi: 10.1007/BF00686619

16. Duyckaerts C, Delaère $P$, Hauw J-J (1992) Alzheimer's disease and neuroanatomy: Hypotheses and proposals. In: Heterogeneity of Alzheimer's disease. Springer, pp 144155

17. Eskelinen E-L, Saftig P (2009) Autophagy: a lysosomal degradation pathway with a central role in health and disease. Biochim Biophys Acta (BBA)-Molecular Cell Res 1793:664-673. doi: 10.1016/j.bbamcr.2008.07.014

18. Funk KE, Mrak RE, Kuret J (2011) Granulovacuolar degeneration (GVD) bodies of Alzheimer's disease $(A D)$ resemble late-stage autophagic organelles. Neuropathol Appl Neurobiol 37:295-306. doi: 10.1111/j.1365-2990.2010.01135.x

19. Ganz AB, Beker N, Hulsman M, Sikkes S, Bank NB, Scheltens P, Smit AB, Rozemuller AJM, Hoozemans JJM, Holstege $H$ (2018) Neuropathology and cognitive performance in self-reported cognitively healthy centenarians. Acta Neuropathol Commun 6:64. doi: 10.1186/s40478-018-0558-5

20. Giacobini E, Gold G (2013) Alzheimer disease therapy-moving from amyloid- $\beta$ to tau. Nat Rev Neurol 9:677. doi: 10.1038/nrneurol.2013.223

21. Goodpaster T, Randolph-Habecker J (2014) A Flexible Mouse-On-Mouse Immunohistochemical Staining Technique Adaptable to Biotin-Free Reagents, Immunofluorescence, and Multiple Antibody Staining. J Histochem Cytochem 62:197204. doi: $10.1369 / 0022155413511620$ 
22. Grootjans S, Vanden Berghe T, Vandenabeele P (2017) Initiation and execution mechanisms of necroptosis: An overview. Cell Death Differ 24:1184-1195. doi: 10.1038/cdd.2017.65

23. He S, Wang L, Miao L, Wang T, Du F, Zhao L, Wang X (2009) Receptor interacting protein kinase-3 determines cellular necrotic response to TNF- $\alpha$. Cell 137:1100-1111. doi: 10.1016/j.cell.2009.05.021

24. Heneka MT, Carson MJ, Khoury J El, Landreth GE, Brosseron F, Feinstein DL, Jacobs AH, Wyss-Coray T, Vitorica J, Ransohoff RM, Herrup K, Frautschy SA, Finsen B, Brown GC, Verkhratsky A, Yamanaka K, Koistinaho J, Latz E, Halle A, Petzold GC, Town T, Morgan D, Shinohara ML, Perry VH, Holmes C, Bazan NG, Brooks DJ, Hunot S, Joseph B, Deigendesch N, Garaschuk O, Boddeke E, Dinarello CA, Breitner JC, Cole GM, Golenbock DT, Kummer MP (2015) Neuroinflammation in Alzheimer's disease. Lancet Neurol 14:388-405. doi: 10.1016/S1474-4422(15)70016-5

25. Hildebrand JM, Tanzer MC, Lucet IS, Young SN, Spall SK, Sharma P, Pierotti C, Garnier J-M, Dobson RCJ, Webb Al (2014) Activation of the pseudokinase MLKL unleashes the four-helix bundle domain to induce membrane localization and necroptotic cell death. Proc Natl Acad Sci 111:15072-15077. doi: 10.1073/pnas.1408987111

26. Hirano A, Dembitzer HM, Kurland LT, Zimmerman HM (1968) The fine structure of some intraganglionic alterations: neurofibrillary tangles, granulovacuolar bodies and "rod-like" structures as seen in Guam amyotrophic lateral sclerosis and parkinsonism-dementia complex. J Neuropathol Exp Neurol 27:167-182. doi: 10.1097/00005072-19680400000001

27. Holmes C, Cunningham C, Zotova E, Woolford J, Dean C, Kerr S ua, Culliford D, Perry $\mathrm{VH}$ (2009) Systemic inflammation and disease progression in Alzheimer disease. Neurology 73:768-774. doi: 10.1212/WNL.0b013e3181b6bb95

28. Hoozemans JJM, van Haastert ES, Nijholt DAT, Rozemuller AJM, Eikelenboom P, Scheper W (2009) The unfolded protein response is activated in pretangle neurons in Alzheimer's disease hippocampus. Am J Pathol 174:1241-1251. doi: 10.2353/ajpath.2009.080814

29. Hyman BT, Van Hoesen GW, Damasio AR, Barnes CL (1984) Alzheimer's disease: cellspecific pathology isolates the hippocampal formation. Science 225:1168-1170. doi: 10.1126/science.6474172

30. Hyman BT, Phelps CH, Beach TG, Bigio EH, Cairns NJ, Carrillo MC, Dickson DW, Duyckaerts C, Frosch MP, Masliah E (2012) National Institute on Aging-Alzheimer's Association guidelines for the neuropathologic assessment of Alzheimer's disease. Alzheimer's Dement 8:1-13. doi: 10.1016/j.jalz.2011.10.007

31. Ito Y, Ofengeim D, Najafov A, Das S, Saberi S, Li Y, Hitomi J, Zhu H, Chen H, Mayo L 
(2016) RIPK1 mediates axonal degeneration by promoting inflammation and necroptosis in ALS. Science 353:603-608. doi: 10.1126/science.aaf6803

32. Jing Z, Caltagarone J, Bowser R (2009) Altered subcellular distribution of c-Abl in Alzheimer's disease. J Alzheimer's Dis 17:409-422. doi: 10.3233/JAD-2009-1062

33. Kadokura A, Yamazaki T, Kakuda S, Makioka K, Lemere CA, Fujita Y, Takatama M, Okamoto K (2009) Phosphorylation-dependent TDP-43 antibody detects intraneuronal dot-like structures showing morphological characters of granulovacuolar degeneration. Neurosci Lett 463:87-92. doi: 10.1016/j.neulet.2009.06.024

34. Kannanayakal TJ, Tao H, Vandre DD, Kuret J (2006) Casein kinase-1 isoforms differentially associate with neurofibrillary and granulovacuolar degeneration lesions. Acta Neuropathol 111:413-421. doi: 10.1007/s00401-006-0049-9

35. Köhler C (2016) Granulovacuolar degeneration: a neurodegenerative change that accompanies tau pathology. Acta Neuropathol 132:339-359. doi: 10.1007/s00401-0161562-0

36. Köhler C, Dinekov M, Götz J (2014) Granulovacuolar degeneration and unfolded protein response in mouse models of tauopathy and $A \beta$ amyloidosis. Neurobiol Dis 71:169179. doi: 10.1016/j.nbd.2014.07.006

37. Kumar S, Wirths O, Stüber K, Wunderlich P, Koch P, Theil S, Rezaei-Ghaleh N, Zweckstetter M, Bayer TA, Brüstle O, Thal DR, Walter J (2016) Phosphorylation of the amyloid $\beta$-peptide at Ser26 stabilizes oligomeric assembly and increases neurotoxicity. Acta Neuropathol 131:525-537. doi: 10.1007/s00401-016-1546-0

38. Lagalwar S, Guillozet-Bongaarts AL, Berry RW, Binder LI (2006) Formation of phosphoSAPK/JNK granules in the hippocampus is an early event in Alzheimer disease. $J$ Neuropathol Exp Neurol 65:455-464. doi: 10.1097/01.jnen.0000229236.98124.d8

39. Leroy K, Boutajangout A, Authelet M, Woodgett JR, Anderton BH, Brion J-P (2002) The active form of glycogen synthase kinase-3 $\beta$ is associated with granulovacuolar degeneration in neurons in Alzheimer's disease. Acta Neuropathol 103:91-99

40. Lewis J, Dickson DW, Lin W-L, Chisholm L, Corral A, Jones G, Yen S-H, Sahara N, Skipper L, Yager D (2001) Enhanced neurofibrillary degeneration in transgenic mice expressing mutant tau and APP. Science 293:1487-1491. doi: 10.1126/science. 1058189

41. Liu S, Liu H, Johnston A, Hanna-Addams S, Reynoso E, Xiang Y, Wang Z (2017) MLKL forms disulfide bond-dependent amyloid-like polymers to induce necroptosis. Proc Natl Acad Sci 114:E7450-E7459. doi: 10.1073/pnas.1707531114

42. Lockshin RA, Zakeri Z (2001) Programmed cell death and apoptosis: origins of the theory. Nat Rev Mol cell Biol 2:545. doi: 10.1038/35080097

43. Lund H, Gustafsson E, Svensson A, Nilsson M, Berg M, Sunnemark D, von Euler G 
(2014) MARK4 and MARK3 associate with early tau phosphorylation in Alzheimer's disease granulovacuolar degeneration bodies. Acta Neuropathol Commun 2:22. doi: 10.1186/2051-5960-2-22

44. Mirra SS, Heyman A, McKeel D, Sumi SM, Crain BJ, Brownlee LM, Vogel FS, Hughes JP, Van Belle G, Berg L (1991) The Consortium to Establish a Registry for Alzheimer's Disease (CERAD): Part II. Standardization of the neuropathologic assessment of Alzheimer's disease. Neurology 41:479. doi: 10.1212/WNL.41.4.479

45. Morris JC, Heyman A, Mohs RC, Hughes JP, Van Belle G, Fillenbaum G, Mellits ED, Clark C (1989) The consortium to establish a registry for Alzheimer's disease (CERAD): I. Clinical and neuropsychological assessment of Alzheimer's disease. Neurology. doi: 10.1212/WNL.39.9.1159

46. Morsch R, Simon W, Coleman PD (1999) Neurons may live for decades with neurofibrillary tangles. J Neuropathol Exp Neurol 58:188-197. doi: 110.1097/00005072199902000-00008

47. Murphy JM, Czabotar PE, Hildebrand JM, Lucet IS, Zhang J-G, Alvarez-Diaz S, Lewis R, Lalaoui N, Metcalf D, Webb Al (2013) The pseudokinase MLKL mediates necroptosis via a molecular switch mechanism. Immunity 39:443-453. doi: 10.1016/j.immuni.2013.06.018

48. Nelson PT, Alafuzoff I, Bigio EH, Bouras C, Braak H, Cairns NJ, Castellani RJ, Crain BJ, Davies P, Tredici K Del (2012) Correlation of Alzheimer disease neuropathologic changes with cognitive status: a review of the literature. J Neuropathol Exp Neurol 71:362-381. doi: 10.1097/NEN.0b013e31825018f7

49. Nishikawa T, Takahashi T, Nakamori M, Hosomi N, Maruyama H, Miyazaki Y, Izumi Y, Matsumoto M (2016) The identification of raft-derived tau-associated vesicles that are incorporated into immature tangles and paired helical filaments. Neuropathol Appl Neurobiol 42:639-653. doi: 10.1111/nan.12288

50. Ofengeim D, Ito Y, Najafov A, Zhang Y, Shan B, DeWitt JP, Ye J, Zhang X, Chang A, Vakifahmetoglu-Norberg $H$ (2015) Activation of necroptosis in multiple sclerosis. Cell Rep 10:1836-1849. doi: 10.1016/j.celrep.2015.02.051

51. Ofengeim D, Mazzitelli S, Ito Y, DeWitt JP, Mifflin L, Zou C, Das S, Adiconis X, Chen H, Zhu H, Kelliher MA, Levin JZ, Yuan J (2017) RIPK1 mediates a disease-associated microglial response in Alzheimer's disease. Proc Natl Acad Sci 201714175. doi: 10.1073/pnas.1714175114

52. Ohm TG, Müller H, Braak H, Bohl J (1995) Close-meshed prevalence rates of different stages as a tool to uncover the rate of Alzheimer's disease-related neurofibrillary changes. Neuroscience 64:209-217. doi: 10.1016/0306-4522(95)90397-P

53. Okamoto K, Hirai S, lizuka T, Yanagisawa T, Watanabe M (1991) Reexamination of 
granulovacuolar degeneration. Acta Neuropathol 82:340-345. doi: 10.1007/BF00296544

54. Pini L, Pievani M, Bocchetta M, Altomare D, Bosco P, Cavedo E, Galluzzi S, Marizzoni M, Frisoni GB (2016) Brain atrophy in Alzheimer's disease and aging. Ageing Res Rev 30:25-48. doi: 10.1016/j.arr.2016.01.002

55. Re DB, Le Verche V, Yu C, Amoroso MW, Politi KA, Phani S, Ikiz B, Hoffmann L, Koolen M, Nagata T (2014) Necroptosis drives motor neuron death in models of both sporadic and familial ALS. Neuron 81:1001-1008. doi: 10.1016/j.neuron.2014.01.011

56. Rijal Upadhaya A, Kosterin I, Kumar S, Von Arnim CAF, Yamaguchi H, Fändrich M, Walter J, Thal DR (2014) Biochemical stages of amyloid- $\beta$ peptide aggregation and accumulation in the human brain and their association with symptomatic and pathologically preclinical Alzheimer's disease. Brain 137:887-903. doi: 10.1093/brain/awt362

57. Riku Y, Duyckaerts C, Boluda S, Plu I, Le Ber I, Millecamps S, Salachas F, Network BNN, Yoshida M, Ando T (2019) Increased prevalence of granulovacuolar degeneration in C9orf72 mutation. Acta Neuropathol 1-11. doi: 10.1007/s00401-019-02028-6

58. Ros U, Peña-Blanco A, Hänggi K, Kunzendorf U, Krautwald S, Wong WWL, GarcíaSáez AJ (2017) Necroptosis Execution Is Mediated by Plasma Membrane Nanopores Independent of Calcium. Cell Rep 19:175-187. doi: 10.1016/j.celrep.2017.03.024

59. Rossi S, Motta C, Studer V, Barbieri F, Buttari F, Bergami A, Sancesario G, Bernardini S, De Angelis G, Martino G (2014) Tumor necrosis factor is elevated in progressive multiple sclerosis and causes excitotoxic neurodegeneration. Mult Scler J 20:304-312. doi: $10.1177 / 1352458513498128$

60. Sassin I, Schultz C, Thal DR, Rüb U, Arai K, Braak E, Braak H (2000) Evolution of Alzheimer's disease-related cytoskeletal changes in the basal nucleus of Meynert. Acta Neuropathol 100:259-269. doi: 10.1007/s004019900178

61. Scheff SW, Price DA, Schmitt FA, Mufson EJ (2006) Hippocampal synaptic loss in early Alzheimer's disease and mild cognitive impairment. Neurobiol Aging 27:1372-1384. doi: 10.1016/j.neurobiolaging.2005.09.012

62. Schwab C, DeMaggio AJ, Ghoshal N, Binder LI, Kuret J, McGeer PL (2000) Casein kinase 1 delta is associated with pathological accumulation of tau in several neurodegenerative diseases. Neurobiol Aging 21:503-510. doi: 10.1016/S01974580(00)00110-X

63. Selznick LA, Holtzman DM, Han BH, Gökden M, Srinivasan AN, Johnson Jr EM, Roth KA (1999) In situ immunodetection of neuronal caspase-3 activation in Alzheimer disease. J Neuropathol Exp Neurol 58:1020-1026. doi: 10.1097/00005072-19990900000012 
64. Simchowicz T (1911) Histologische und histopathologische Arbeiten über die Grosshirnrinde mit besonderer Berücksichtigung der pathologischen Anatomie der Geisteskrankheiten Histologische Studien über die senile Demenz

65. Stadelmann C, Deckwerth TL, Srinivasan A, Bancher C, Brück W, Jellinger K, Lassmann $H$ (1999) Activation of caspase-3 in single neurons and autophagic granules of granulovacuolar degeneration in Alzheimer's disease: evidence for apoptotic cell death. Am J Pathol 155:1459-1466. doi: 10.1016/S0002-9440(10)65460-0

66. Stadelmann C, Lassmann H (2000) Detection of apoptosis in tissue sections. Cell Tissue Res 301:19-31. doi: 10.1007/s004410000203

67. Su JH, Kesslak PJ, Head E, Cotman CW (2002) Caspase-cleaved amyloid precursor protein and activated caspase-3 are co-localized in the granules of granulovacuolar degeneration in Alzheimer's disease and Down's syndrome brain. Acta Neuropathol 104:1-6. doi: 10.1007/s00401-002-0548-2

68. Sun L, Wang H, Wang Z, He S, Chen S, Liao D, Wang L, Yan J, Liu W, Lei X (2012) Mixed lineage kinase domain-like protein mediates necrosis signaling downstream of RIP3 kinase. Cell 148:213-227. doi: 10.1016/j.cell.2011.11.031

69. Susaki EA, Tainaka K, Perrin D, Yukinaga H, Kuno A, Ueda HR (2015) Advanced CUBIC protocols for whole-brain and whole-body clearing and imaging. Nat Protoc 10:1709. doi: 10.1038/nprot.2015.085

70. Terry RD, DeTeresa R, Hansen LA (1987) Neocortical cell counts in normal human adult aging. Ann Neurol Off J Am Neurol Assoc Child Neurol Soc 21:530-539. doi: 10.1002/ana.410210603

71. Thal DR, Holzer M, Rüb U, Waldmann G, Günzel S, Zedlick D, Schober R (2000) Alzheimer-related T-pathology in the perforant path target zone and in the hippocampal stratum oriens and radiatum correlates with onset and degree of dementia. Exp Neurol 163:98-110. doi: 10.1006/exnr.2000.7380

72. Thal DR, Rüb $U$, Orantes $M$, Braak $H$ (2002) Phases of $A \beta$-deposition in the human brain and its relevance for the development of AD. Neurology 58:1791-1800. doi: 10.1212/WNL.58.12.1791

73. Thal DR, Rüb U, Schultz C, Sassin I, Ghebremedhin E, Del Tredici K, Braak E, Braak $\mathrm{H}(2000)$ Sequence of $A \beta$-protein deposition in the human medial temporal lobe. $J$ Neuropathol Exp Neurol 59:733-748. doi: 10.1093/jnen/59.8.733

74. Thal DR, Del Tredici K, Ludolph AC, Hoozemans JJM, Rozemuller AJ, Braak H, Knippschild U (2011) Stages of granulovacuolar degeneration: Their relation to Alzheimer's disease and chronic stress response. Acta Neuropathol 122:577-589. doi: 10.1007/s00401-011-0871-6

75. Tomlinson BE, Kitchener D (1972) Granulovacuolar degeneration of hippocampal 
pyramidal cells. J Pathol 106:165-185. doi: 10.1002/path.1711060305

76. Virard F, Cousty S, Cambus J-P, Valentin A, Kémoun P, Clément F (2015) Cold atmospheric plasma induces a predominantly necrotic cell death via the microenvironment. PLoS One 10:e0133120. doi: 10.1371/journal.pone.0133120

77. Wang Y, Martinez-Vicente M, Krüger U, Kaushik S, Wong E, Mandelkow E-M, Cuervo AM, Mandelkow E (2009) Tau fragmentation, aggregation and clearance: the dual role of lysosomal processing. Hum Mol Genet 18:4153-4170. doi: 10.1093/hmg/ddp367

78. Wegner KW, Saleh D, Degterev A (2017) Complex Pathologic Roles of RIPK1 and RIPK3: Moving Beyond Necroptosis. Trends Pharmacol Sci 38:202-225. doi: 10.1016/j.tips.2016.12.005

79. West MJ, Coleman PD, Flood DG, Troncoso JC (1994) Differences in the pattern of hippocampal neuronal loss in normal ageing and Alzheimer's disease. Lancet 344:769772. doi: 10.1016/S0140-6736(94)92338-8

80. Wiersma VI, van Ziel AM, Vazquez-Sanchez S, Nölle A, Berenjeno-Correa E, Bonaterra-Pastra A, Clavaguera F, Tolnay M, Musters RJP, van Weering JRT (2019) Granulovacuolar degeneration bodies are neuron-selective lysosomal structures induced by intracellular tau pathology. Acta Neuropathol 1-28. doi: 10.1007/s00401019-02046-4

81. Yamazaki Y, Matsubara T, Takahashi T, Kurashige T, Dohi E, Hiji M, Nagano Y, Yamawaki T, Matsumoto M (2011) Granulovacuolar degenerations appear in relation to hippocampal phosphorylated tau accumulation in various neurodegenerative disorders. PLoS One 6:e26996. doi: 10.1371/journal.pone.0026996

82. Zarow C, Vinters H V, Ellis WG, Weiner MW, Mungas D, White L, Chui HC (2005) Correlates of hippocampal neuron number in Alzheimer's disease and ischemic vascular dementia. Ann Neurol Off J Am Neurol Assoc Child Neurol Soc 57:896-903. doi: 10.1002/ana.20503

83. Zhang D-W, Shao J, Lin J, Zhang N, Lu B-J, Lin S-C, Dong M-Q, Han J (2009) RIP3, an energy metabolism regulator that switches TNF-induced cell death from apoptosis to necrosis. Science 325:332-336. doi: 10.1126/science.1172308

84. Zhao J, Jitkaew S, Cai Z, Choksi S, Li Q, Luo J, Liu Z-G (2012) Mixed lineage kinase domain-like is a key receptor interacting protein 3 downstream component of TNFinduced necrosis. Proc Natl Acad Sci 109:5322-5327. doi: 10.1073/pnas.1200012109

85. Zlokovic B V (2011) Neurovascular pathways to neurodegeneration in Alzheimer's disease and other disorders. Nat Rev Neurosci 12:723. doi: 10.1038/nrn3114 


\section{Figures}

Fig. 1. Necrosome components are localized in GVD lesions in the $A D$ brain. $D A B$ immunohistochemical stainings for necrosome components ( $p R I P K 1, p R I P K 3, p M L K L)$. AD human tissues revealed a specific immunoreactivity for all three necrosome components, including pRIPK1 (a, d), pRIPK3 (b, e) and pMLKL (c, f), in GVD granules of CA1 hippocampal pyramidal neurons (a-c; red arrowheads), but not in GVD free neurons (a-c; black arrowheads). pRIPK1, pRIPK3 and pMLKL immunoreactivity was detected in the granules and the membranes of the GVD vacuoles (d-f; red arrows), but not in the lumen. Staining of a representative $A D$ case (a-f; Case 3); $n=63$ (non-AD, $n=23$; p-preAD, $n=24 ; A D, n=16$ ). Scale bars represent $30 \mu \mathrm{m}$. Abbreviations: $A D=$ Alzheimer's disease; $C A=$ cornu ammonis.

Fig. 2. pMLKL expression in neuronal GVD lesions. Fluorescent immunohistochemistry for a marker of the activated necrosome, i.e. pMLKL (green; arrowheads), together with markers for microglia (IBA1; red; Mi), astrocytes (GFAP; magenta) and cell nuclei (DAPI, blue) in the $C A 1$ hippocampal region of the non-AD, p-preAD and $A D$ group. In the context of $A D$, pMLKL(S358) was expressed in GVD granules of neurons and not co-expressed with the astrocytic or microglial markers. Staining of representative cases for non-AD (Case 52; $n=4$ ), p-preAD (Case 35; $n=4)$ and $A D($ Case $4 ; n=4)$. Scale bars represent $30 \mu \mathrm{m}$. Abbreviations: $A D=$ Alzheimer's disease; $p$-pre $A D=$ preclinical $A D ;$ non- $A D=$ non-demented control; $C A=$ cornu ammonis; $M i=$ microglia.

Fig. 3. Necrosome markers are co-expressed in GVD granules and colocalize with standard markers for GVD. Representative hippocampal CA1 pyramidal neurons labeled with anti-pRIPK1 (green) and anti-pRIPK3 (red), showing the assembly of the pRIPK1-pRIPK3 necrosome complex in GVD granules in neurons (a-d; arrowheads). In the same region, pRIPK3-expressing neurons (red) are also immunoreactive for pMLKL (green), representing a late phase of necrosome activation with an activated pRIPK3 recruiting and phosphorylating MLKL (e-h; arrowheads). CA1 pyramidal neurons with DAPI stained cell nuclei, depicting colocalization of pMLKL (green) with CK1ס (red; i-l; arrowheads) or with pTDP-43 (red; m-p; arrowheads). Staining of a representative $A D$ case (a-p; Case 4 ); $n=12$ (non-AD, $n=4 ; p$ preAD, $\mathrm{n}=4 ; \mathrm{AD}, \mathrm{n}=4$ ). Scale bars represent $30 \mu \mathrm{m}$. Abbreviations: $A D=$ Alzheimer's disease; $C A=$ cornu ammonis.

Fig. 4. Necrosome-positive GVD stage increases with the progression of AD. GVD stages based on pMLKL-immunoreactivity in GVD lesions in representative brain regions, i.e. CA1/2 and subiculum (stage I), entorhinal cortex and/or CA4, CA3 (stage II), temporal cortex (stage III), amygdala and/or hypothalamus (stage IV), and frontal cortex (stage V). Kruskal-Wallis test with Dunn's multiple comparisons. Data are presented as mean \pm SEM. ${ }^{*} p<0.05 ;{ }^{* *} p<0.01$; 
${ }^{* * *} p<0.001 ;{ }^{* * *} p<0.0001$. Abbreviations: $A D=$ Alzheimer's disease; $p-p r e A D=$ preclinical $A D ;$ non $-A D=$ non-demented control; $C A=$ cornu ammonis.

Fig. 5. GVD-related necrosome activation is associated with Tau but not A $\beta$ pathology. (a-o) Representative images illustrating pMLKL (green), AT8 (red) and 4G8 (magenta) expression pattern and their colocalization in the CA1 hippocampal region of the AD, p-preAD and non-AD group. DAPI was used to stain the nuclei. PMLKL colocalized only with AT8, revealing the association between necroptosis and Tau pathology, but not $A \beta$ pathology. $A D$ $(\mathrm{k}-\mathrm{o})$ and p-preAD (f-j) panels depict co-existence of pMLKL-positive GVD and pTau-positive NFTs. Specifically, three phenotypes of AD-affected neurons are depicted: 1) pMLKL-positive and pTau-negative neurons (o; arrowhead); (2) pMLKL- and pTau-positive neurons (o; arrow); and 3) pTau-positive tangles and pre-tangles in the absence of pMLKL-immunoreactive GVD (j; arrow). Staining of representative cases for non-AD ( $n=4$; Case 52), p-preAD $(n=4$; Case $25)$ and $A D(n=4$; Case 12). ( $p-t)$ Positive immunoreactivity for $4 G 8$ in a neuritic plaque ( $r$; $N P$ ). Staining of a representative $A D$ case (Case 12$) ; n=12$ (non-AD, $n=4 ; p-p r e A D, n=4$; $\mathrm{AD}, \mathrm{n}=4)$. Scale bars represent $30 \mu \mathrm{m}$. Abbreviations: $A D=$ Alzheimer's disease; $p$-pre $A D=$ preclinical $A D ;$ non- $A D=$ non-demented control; $C A=$ cornu ammonis; $N P=$ neuritic plaque.

Fig. 6. GVDn+ neurons correlate with neuronal loss in the hippocampus and frontal cortex. (a, b) Quantitative data representing the percentage of GVDn+ (pMLKL-positive GVDbearing) neurons to the total number of neurons in the hippocampal CA1 region (a) and in the frontal cortex layer III region (b) of the AD, p-preAD and non-AD group. Kruskal-Wallis test followed by Dunn's multiple comparisons. (c, d) In the same regions of interest, quantitative data showing cell density in the $A D, p-p r e A D$ and non-AD group. One-way ANOVA followed by Tukey's multiple comparisons test; $n=63$ (non-AD, $n=16$; p-preAD, $n=24 ; A D, n=23$ ). Data are presented as mean \pm SEM. ${ }^{*} p<0.05 ;{ }^{* *} p<0.01 ;{ }^{* * *} p<0.001 ;{ }^{* * * *} p<0.0001$. Abbreviations: $A D=$ Alzheimer's disease; $p-p r e A D=$ preclinical $A D ;$ non $-A D=$ non-demented control; $C A=$ cornu ammonis.

Fig. 7. Histopathological characterization of non-phosphorylated proteins. DAB immunohistochemical stainings for non-phosphorylated necroptosis-related proteins. (a-d) Positive RIPK1 immunostainings of GVD granules (a-d, red arrows), tangles (c, black arrow) and activated astrocytes (a-c, $A s$ ) were observed in the CA1 region. ( $\mathrm{f}-\mathrm{h}$ ) In the same region, RIPK3-positive cytoplasmic lesions ( $f, g$; gray arrows) and neuritic plaques ( $f, N P$ ) were detected. Using hematoxylin counterstaining, GVD granules remained negative for RIPK3 ( $h$, green arrowheads). Only after dephosphorylation, GVD granules became detectable with antiRIPK3 (see Suppl. Figure 2), confirming the presence of phosphorylated RIPK3 in GVD lesions. Representative images of non-AD control cases for RIPK1 (e), and RIPK3 (i) are depicted. Staining of representative AD (a-d, Case 3; f, Case 12; g, h, Case 13) and non-AD 
cases (e, Case 57; i, Case 54); $n=31$ (non-AD, $n=10 ; p$-preAD, $n=9 ; A D, n=12$ ). Scale bars represent $30 \mu \mathrm{m}$. Abbreviations: $A D=$ Alzheimer's disease non- $A D=$ non-demented control; $A s=$ astrocyte; $C A=$ cornu ammonis; $N P=$ neuritic plaque.

Fig. 8. RIPK1 and RIPK3 expression in different cell types. Fluorescent immunohistochemistry for RIPK1 (a, green) or RIPK3 (b, green) together with markers for microglia (IBA1, red), astrocytes (GFAP, magenta) and cell nuclei (DAPI, blue) in the hippocampal CA1 of the $A D, p-p r e A D$ and non-AD group. (a) Images depict RIPK1immunoreactive cells colocalizing with the astrocyte marker GFAP. The co-expression of RIPK1 with the microglial marker IBA1 was not observed. (b) Immunofluorescence confirmed the expression of RIPK3 in neuronal cytoplasm and not in microglia or astrocytes. Staining of representative cases for non-AD (Case 52; $n=4$ ), p-preAD (Case 35; $n=4$ ) and AD (Case 4; $\mathrm{n}=4$ ). Scale bars represent $30 \mu \mathrm{m}$. Abbreviations: $A D=$ Alzheimer's disease; $p$-pre $A D=$ preclinical $A D ; n o n-A D=$ non-demented control; $C A=$ cornu ammonis.

Fig. 9. Biochemical characterization of necroptosis-related proteins. (a) Temporal cortex samples on western blots probed with RIPK1, pRIPK1 and MLKL antibody. TSZ-treated SHSY5Y cells were used as a positive control and glyceraldehyde 3-phosphate dehydrogenase (GAPDH) as an internal loading control. Full blots are shown in Suppl. Figure 7. (b-d) Quantification of western blot data showing no differences between groups for RIPK1 (b), increased levels of pRIPK1 in the AD cohort compared to controls (c), and increased levels of $M L K L$ in the $A D$ and $p$-preAD group relative to non-AD controls (d). Protein quantifications were normalized to GAPDH. Statistical analyses were performed using one-way ANOVA, followed by Tukey's post-hoc test for multiple comparisons. Data are presented as mean \pm SEM. $A D=$ Cases 1-4, 9; p-preAD = Cases 26, 27, 39, 42, 43; non-AD = Cases 48, 55, 56, 58, 61. Abbreviations: $A D=$ Alzheimer's disease; $p-p r e A D=$ preclinical $A D ;$ non- $A D=$ nondemented control; + = positive control.

\section{Tables}

Table. 1. Cohort characteristics. The table shows age in years, sex, the AßMTL phase representing the distribution of $A \beta$ deposits in the subfields of the MTL [73], the stage of neurofibrillary tangle pathology according to Braak and Braak [9] (NFT stage), the CERAD score for the frequency of neuritic plaques according to Mirra et al. [44], the NIA-AA degree of $A D$ pathology [30], the degree of dementia (CDR score) [45], the pMLKL-GVD stage, neuropathological diagnosis, and PMI. Abbreviations: $f=$ female; $m=$ male; $A D=$ Alzheimer's disease; $p-p r e A D=$ preclinical $A D ;$ non- $A D=$ non-demented control; $A D R P=$ Alzheimer's disease-related metabolic brain pattern; $A G D=$ argyrophilic grain disease, $A R T A G=$ agingrelated tau astrogliopathy; $B=$ bleeding; $C A A=$ cerebrovascular angiopathy; $C D R=$ clinical 
dementia rating; $C E R A D=$ Consortium to Establish a Registry for Alzheimer's disease; $C M=$ carcinoma metastasis; $I$ = infarction; $M I=$ microinfraction; $M C I=$ mild cognitive impairment; $M T L=$ medial temporal lobe; $n . d .=$ not determined; $N F T=$ neurofibrillary tangle; $N I A-A A=$ National Institute on Aging-Alzheimer's Association; $P M I=$ post-mortem interval; $T=$ trauma. 
Figure 1. Necrosome components are localized in GVD lesions in the AD brain

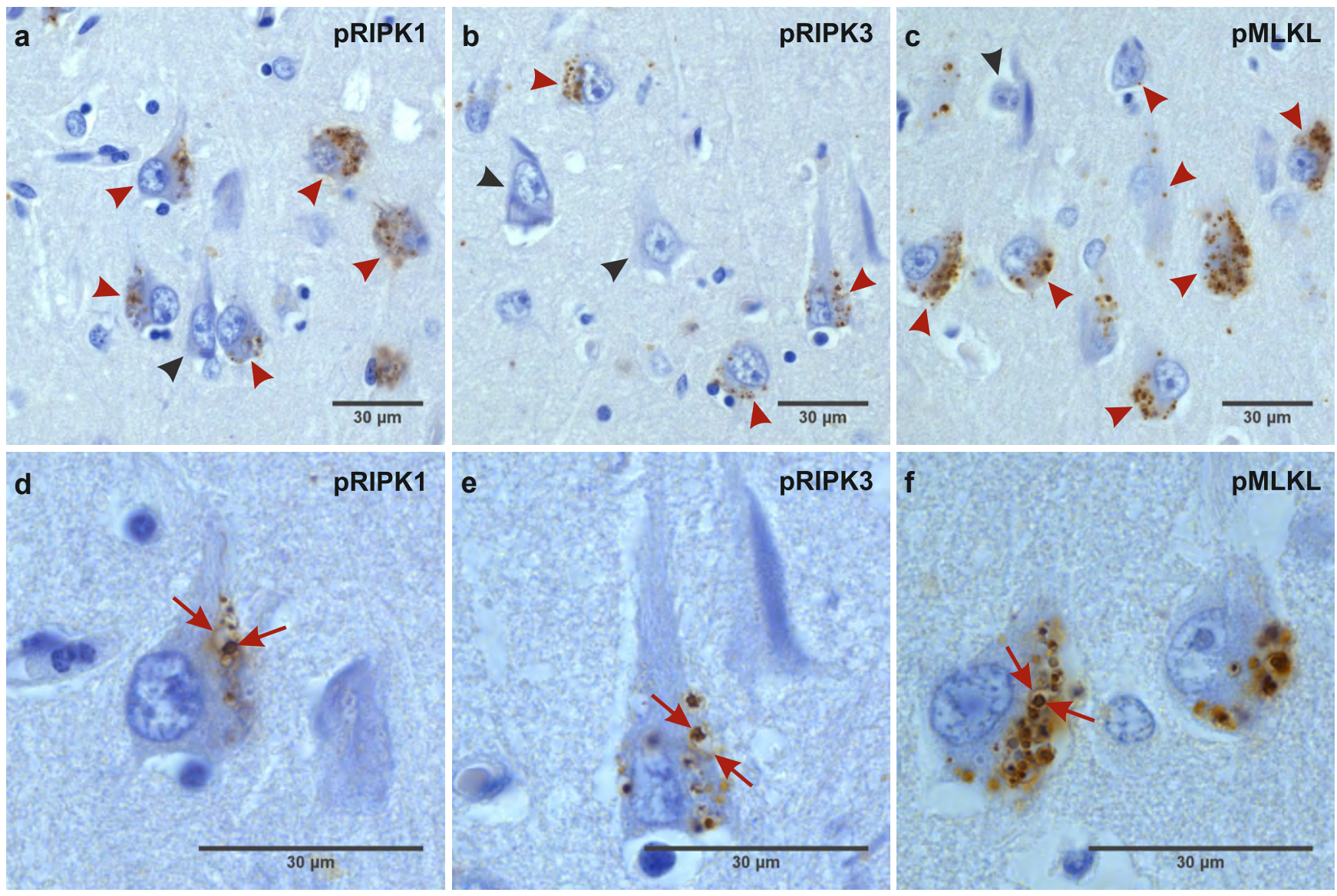


Figure 2. pMLKL expression in neuronal GVD lesions

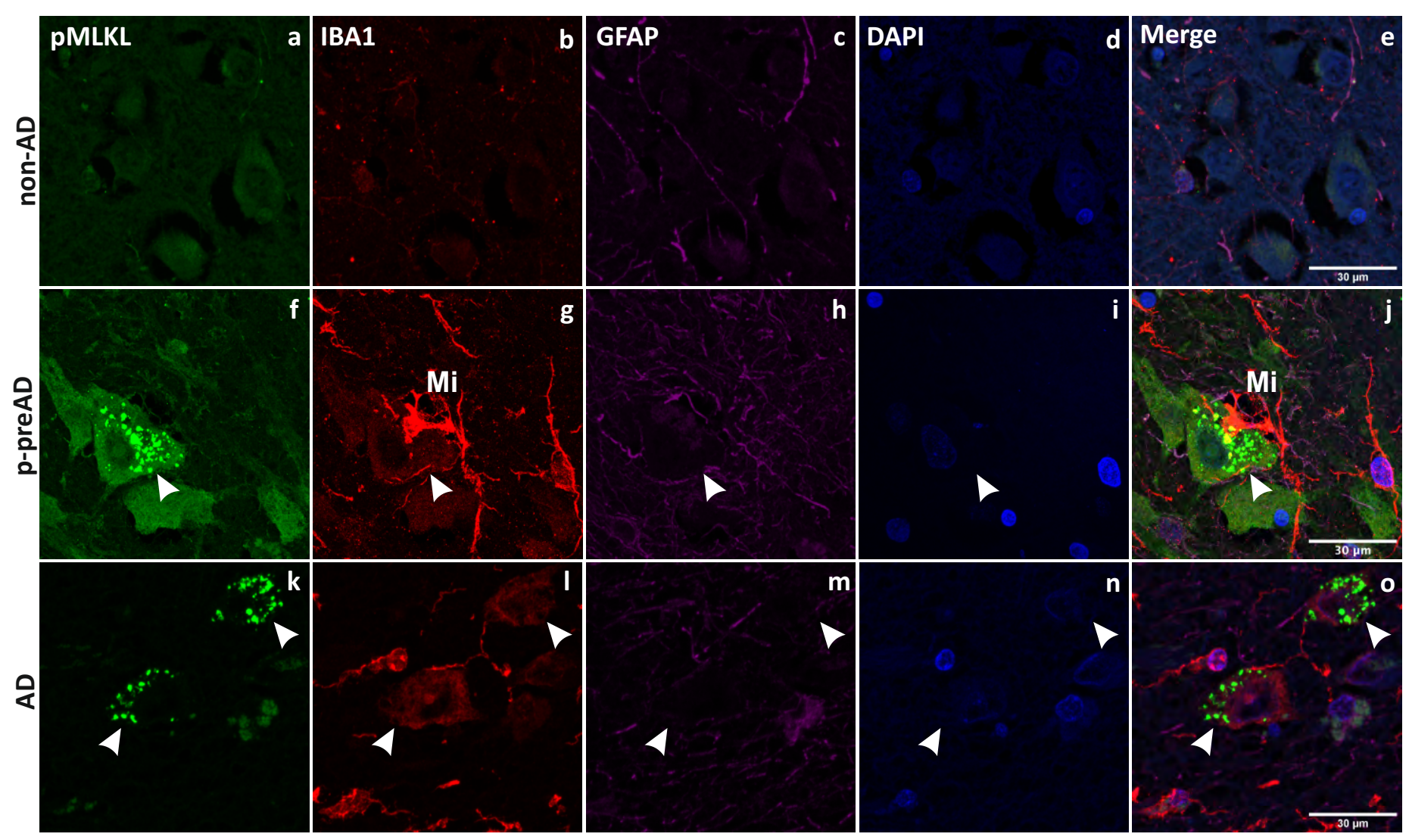


Figure 3. Necrosome markers are co-expressed in GVD granules and colocalize with standard markers for GVD

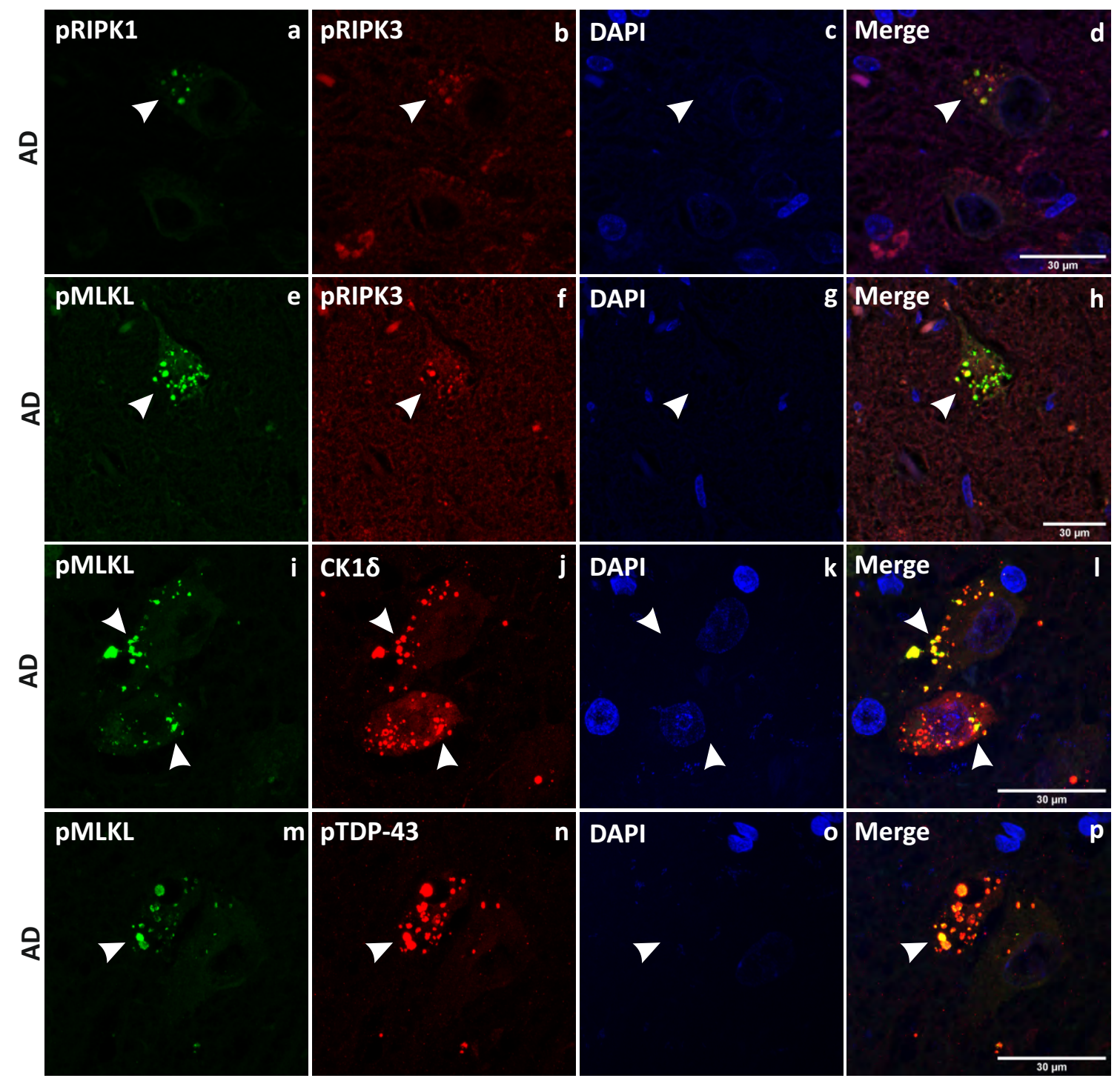


Figure 4. Necrosome-positive GVD stage increases with the progression of AD

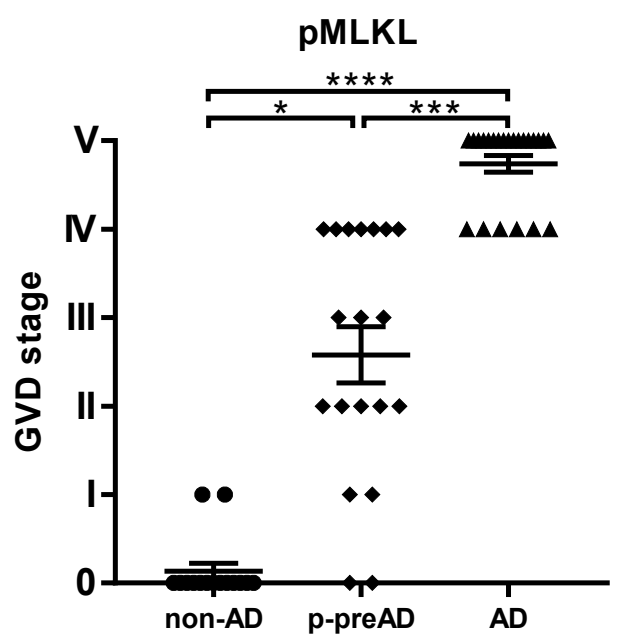


Figure 5. GVD-related necrosome activation is associated with Tau but not A $\beta$ pathology

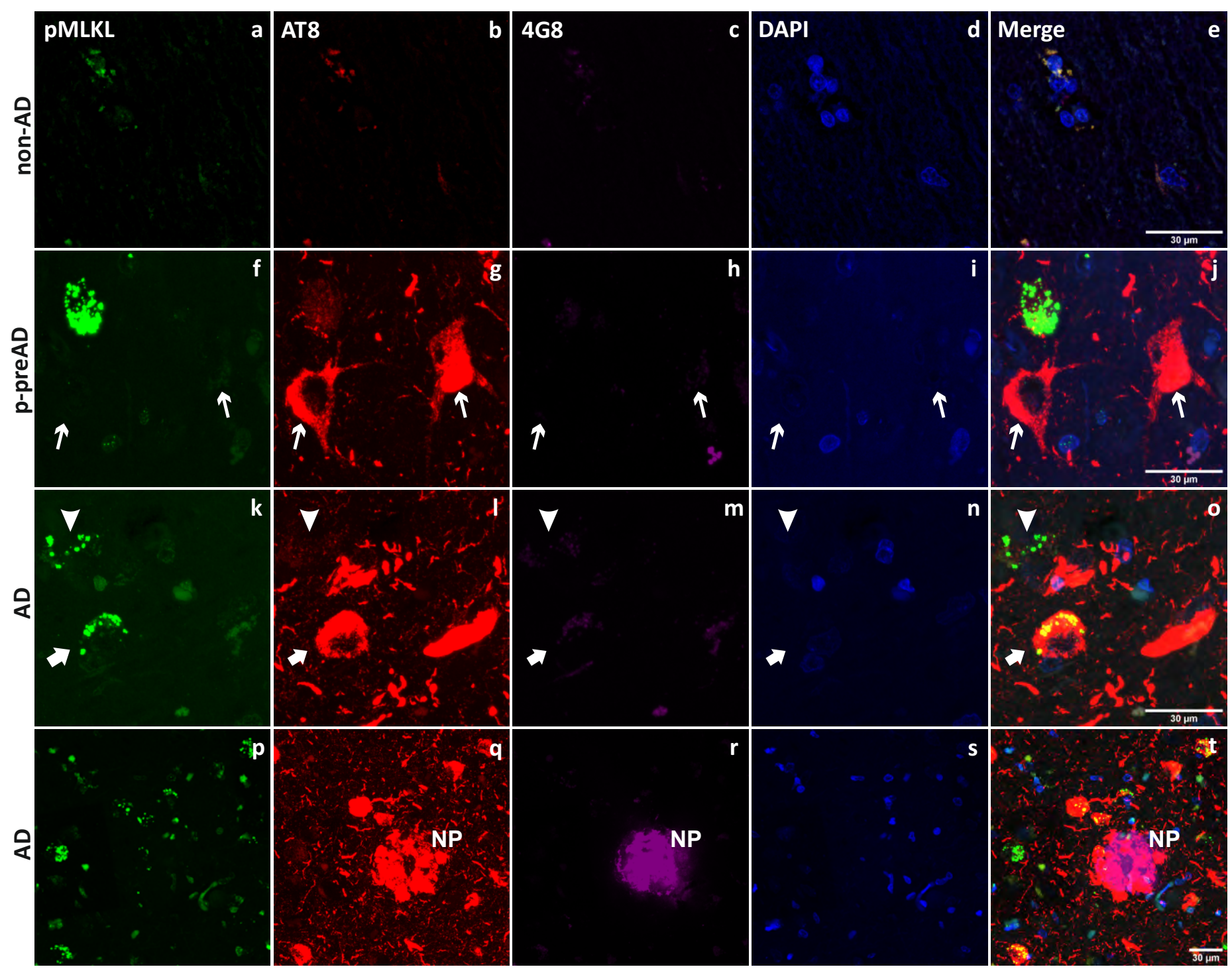


Figure 6. pMLKL expressed in GVD correlates with neuronal loss in the hippocampus and frontal cortex

a

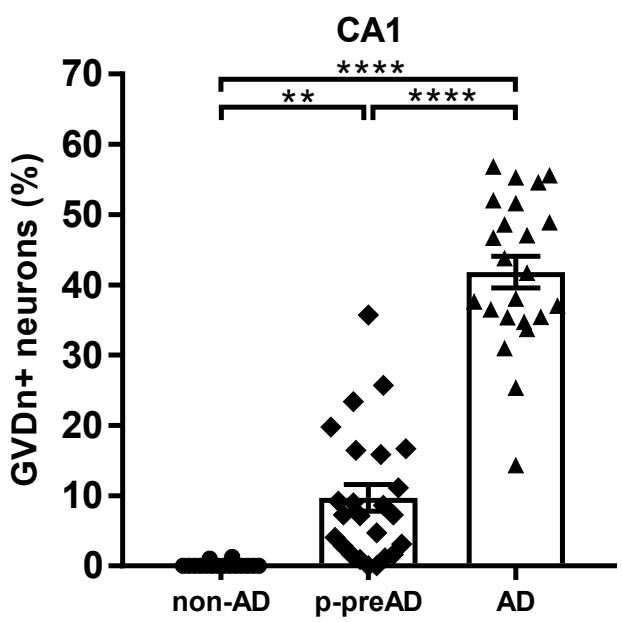

C

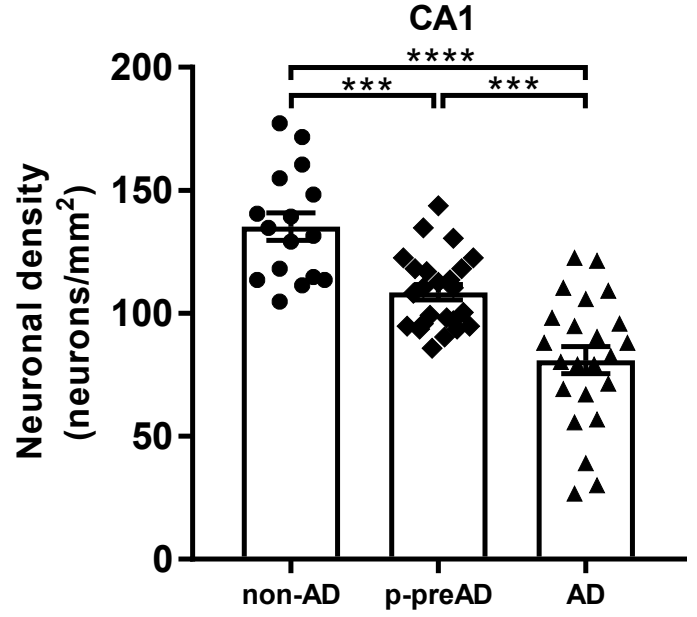

b

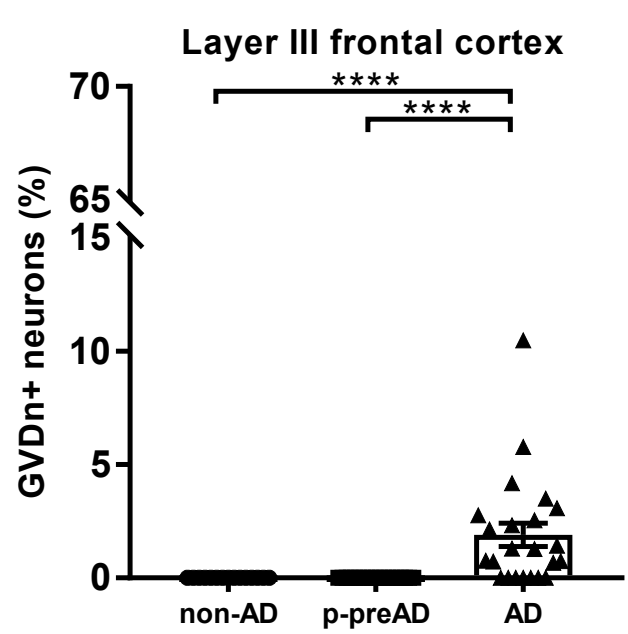

d

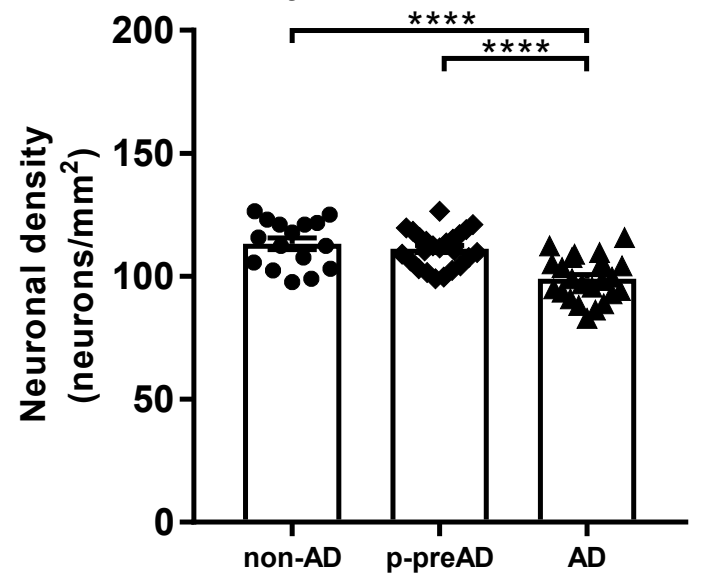


Figure 7. Histopathological characterization of non-phosphorylated proteins

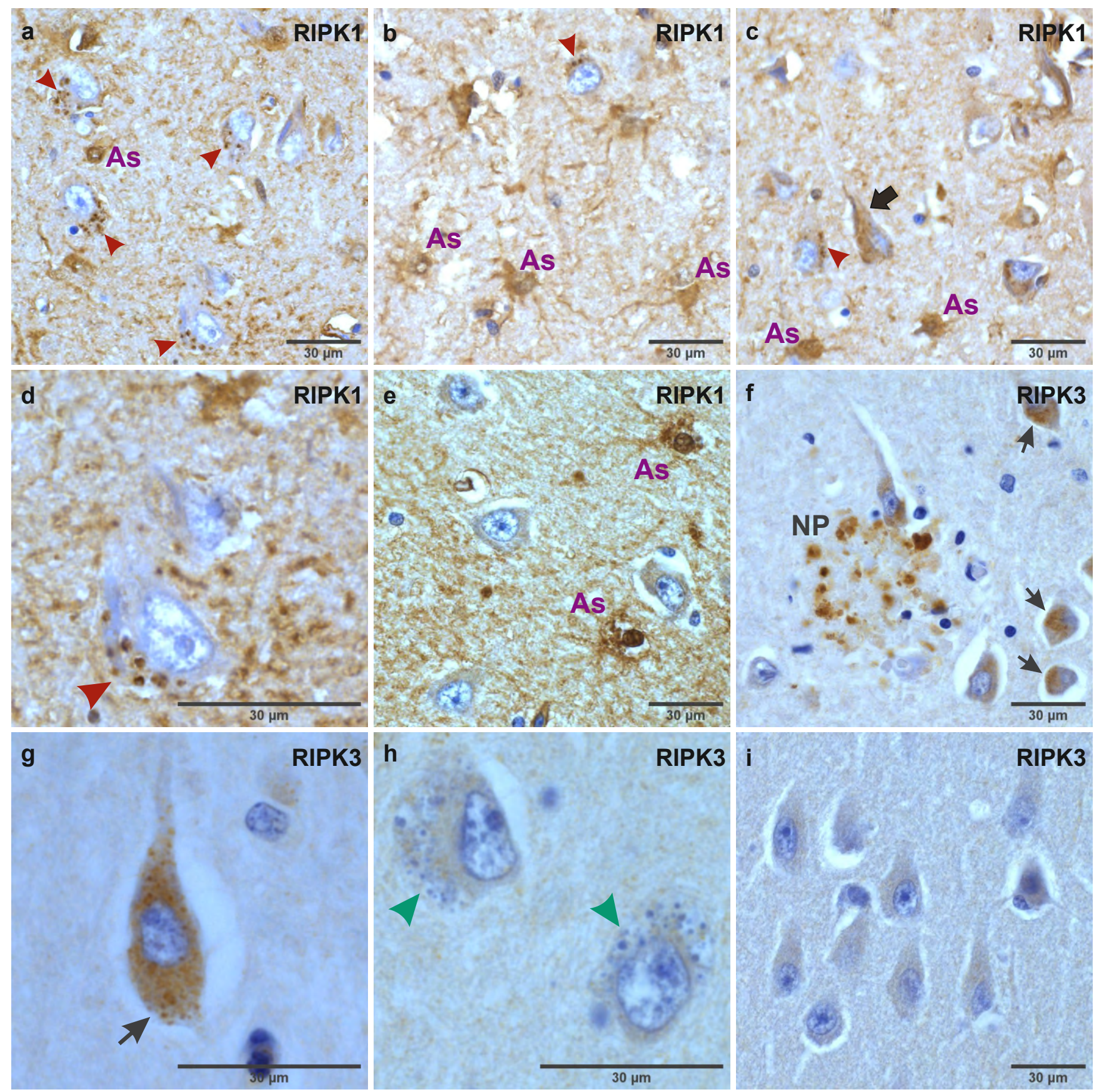


Figure 8. RIPK1 and RIPK3 expression in different cell types

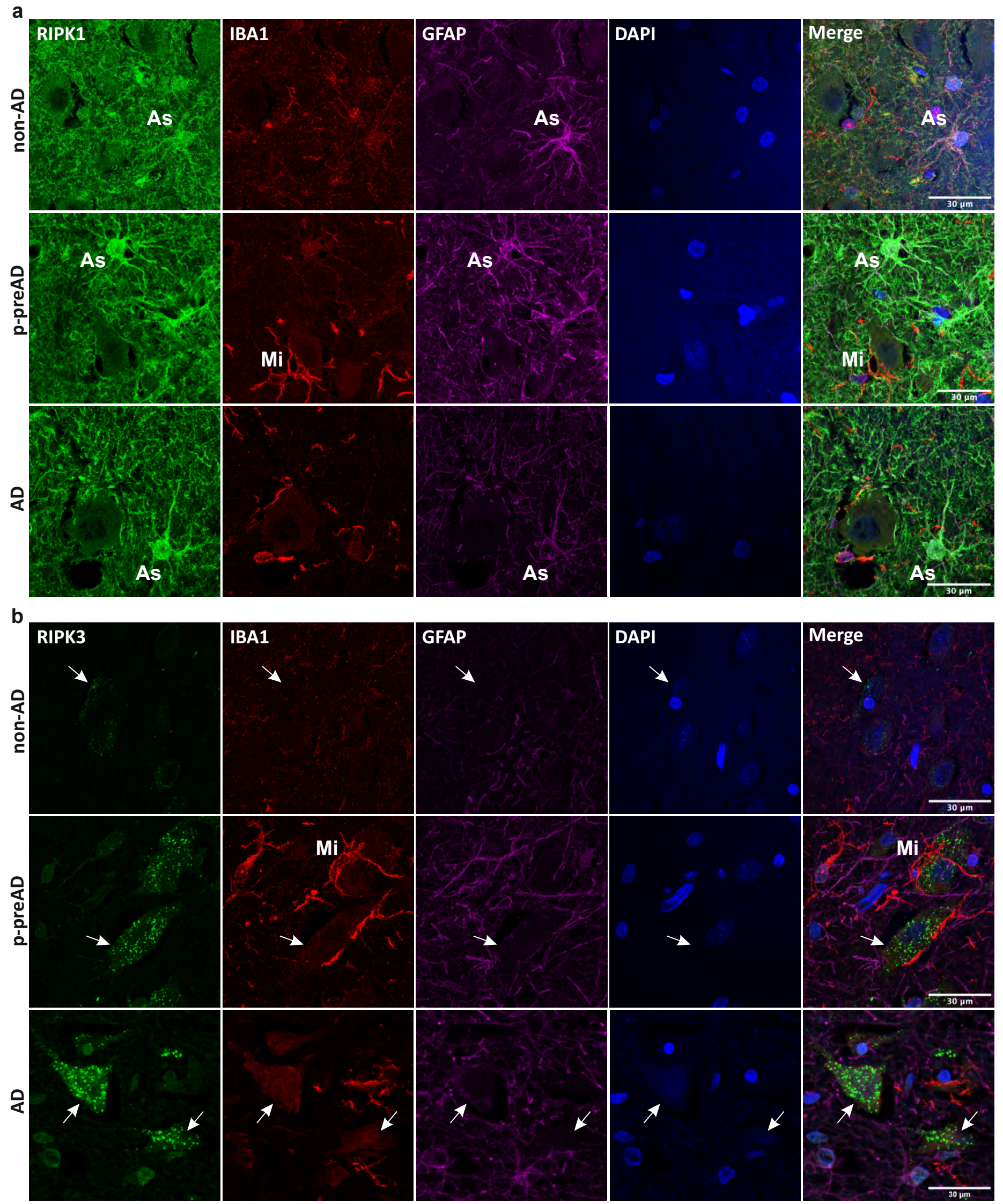


Figure 9. Biochemical characterization of necroptosis-related proteins

a

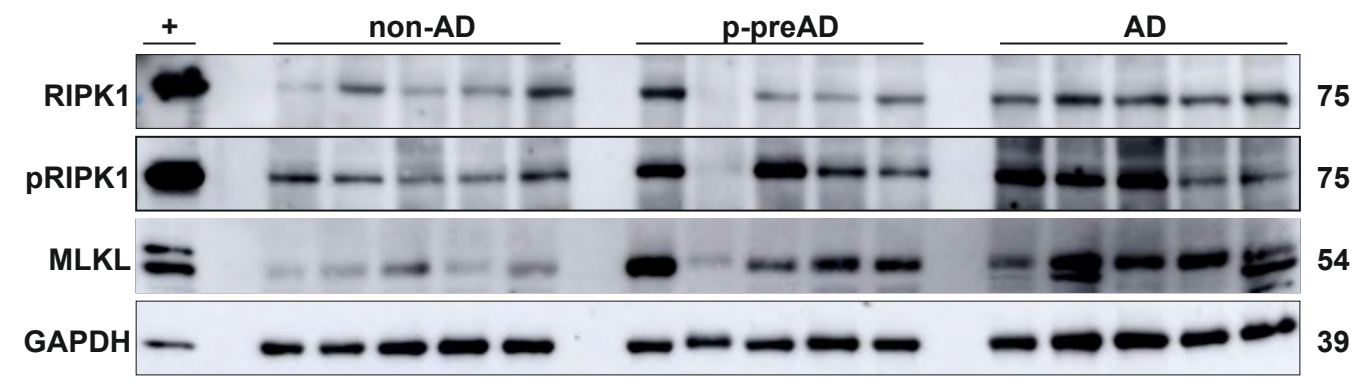

b

RIPK1

c

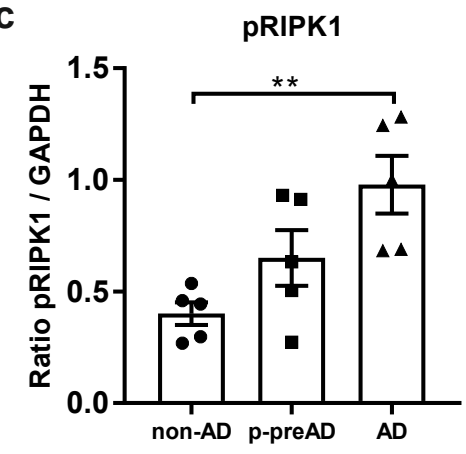

d MLKL
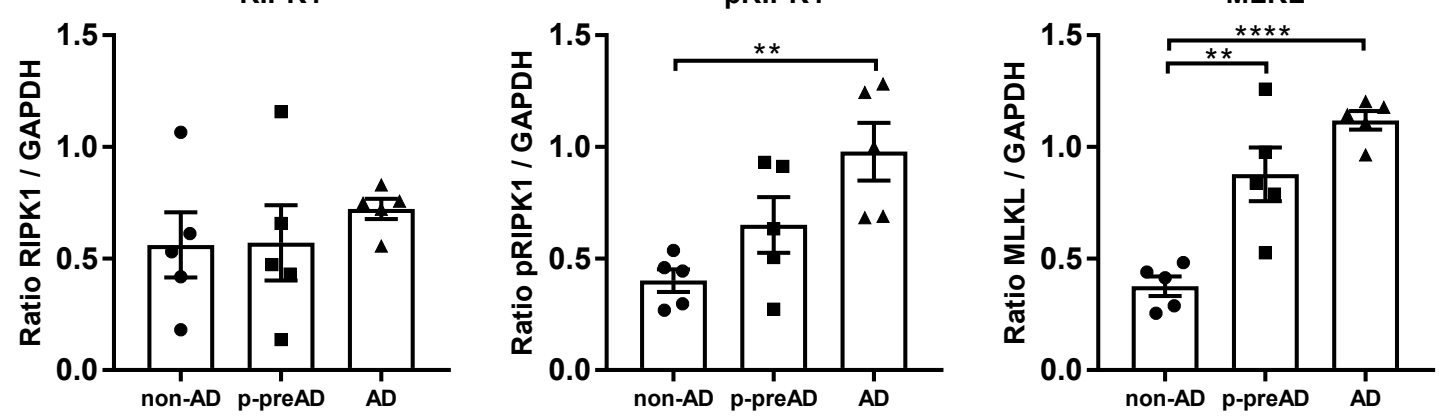
Suppl. Figure 1. Determination of the specificity of necroptosis antibodies against pRIPK1, pRIPK3 and pMLKL using blocking peptides

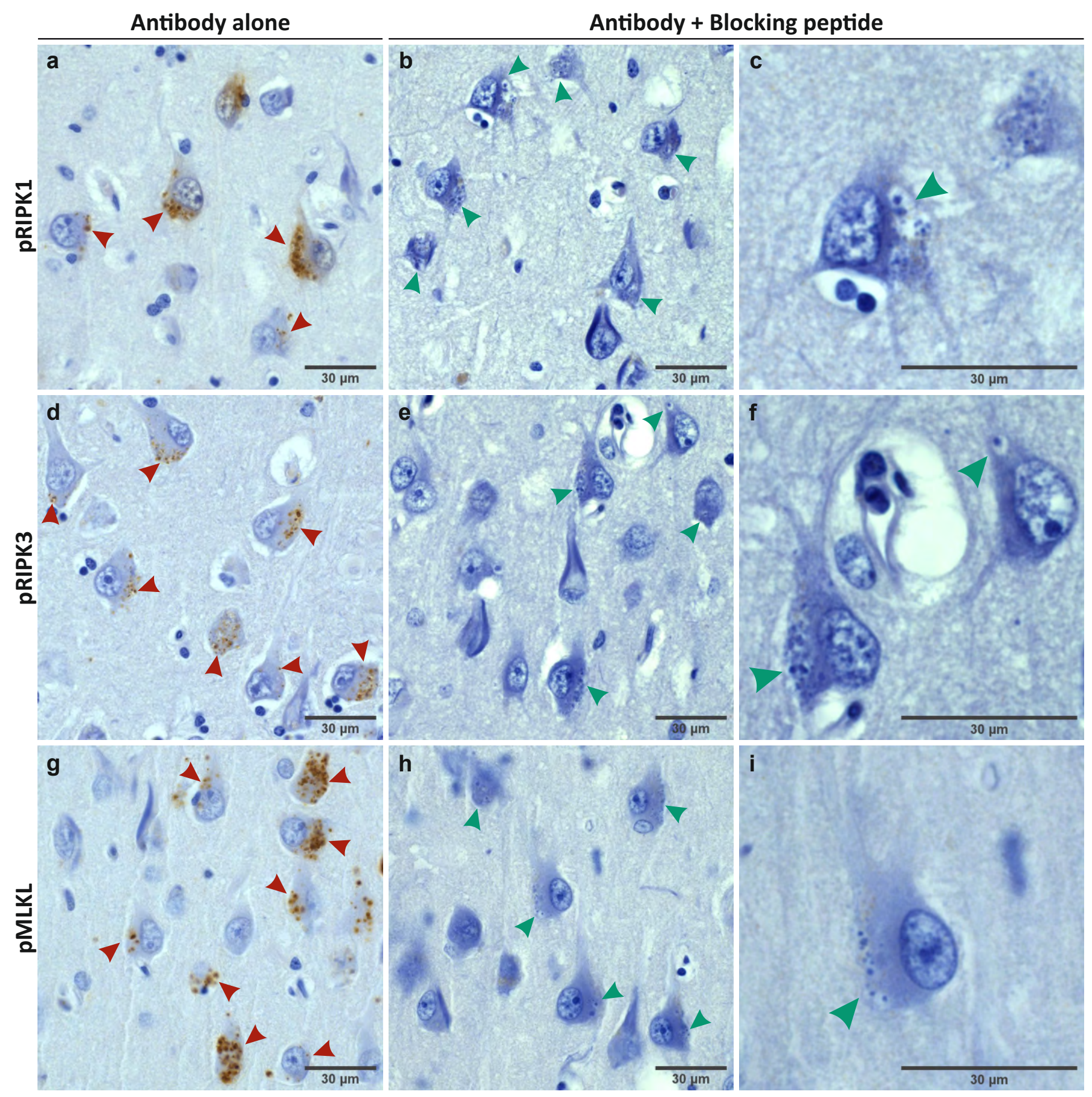


Suppl. Figure 2. Determination of the specificity of necroptosis antibodies against pRIPK1, pRIPK3 and pMLKL on dephosphorylated tissue

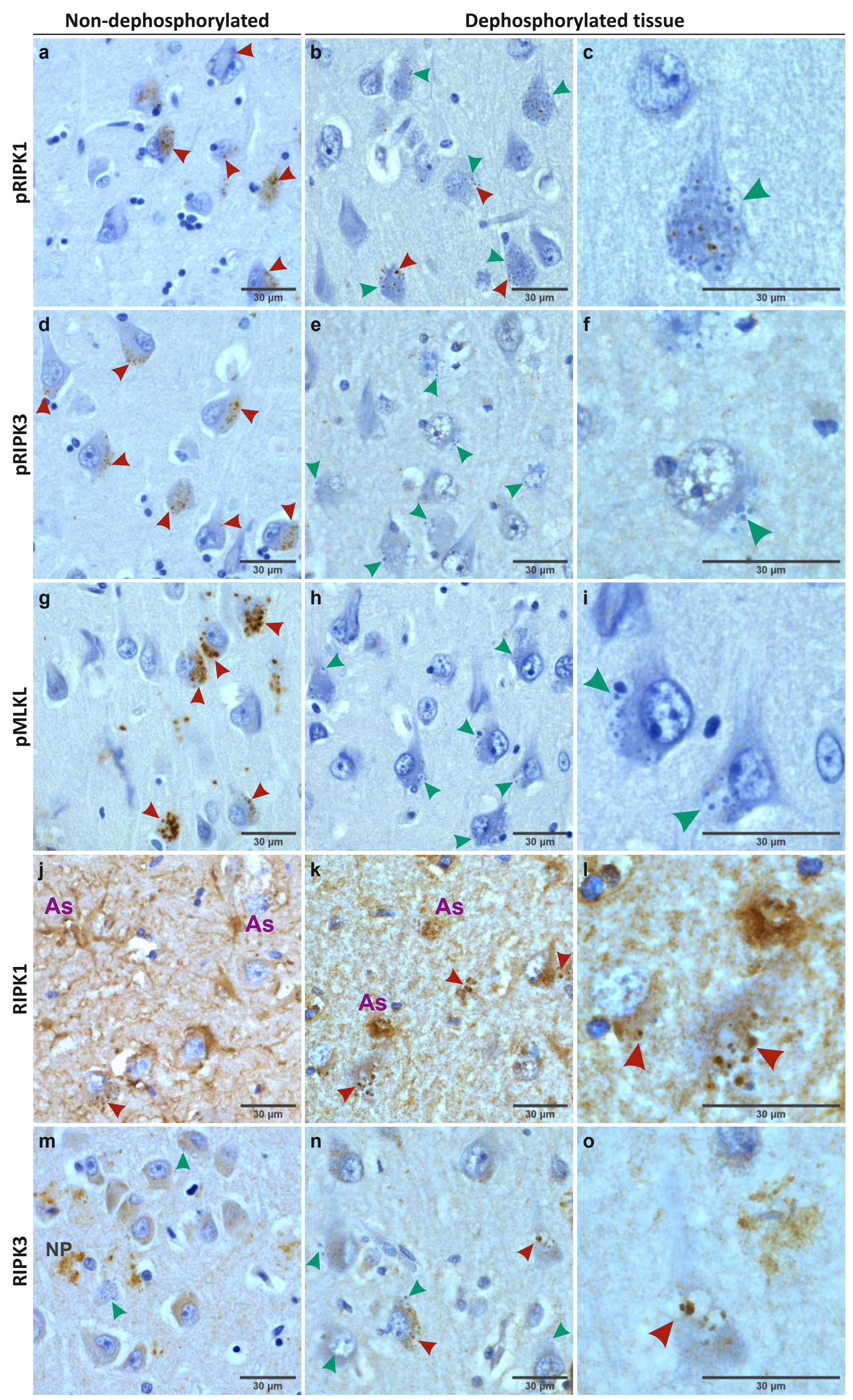


Suppl. Figure 3. Validation of the presence of pMLKL in GVD inclusions by using a second antibody against $\mathrm{PMLKL}$

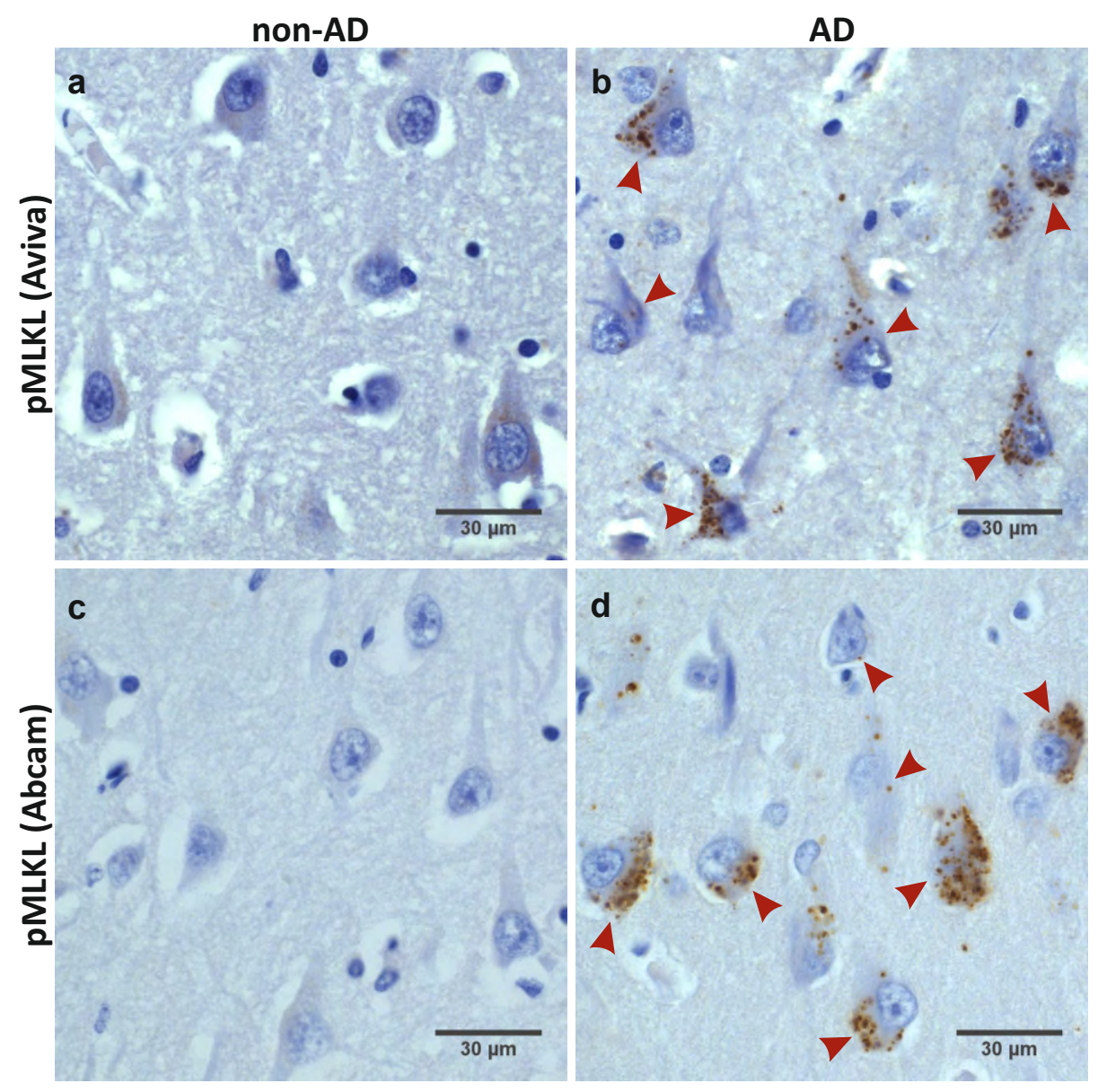


Suppl. Figure 4. Semi-quantitative analysis of pMLKL expression in the MTL regions

a

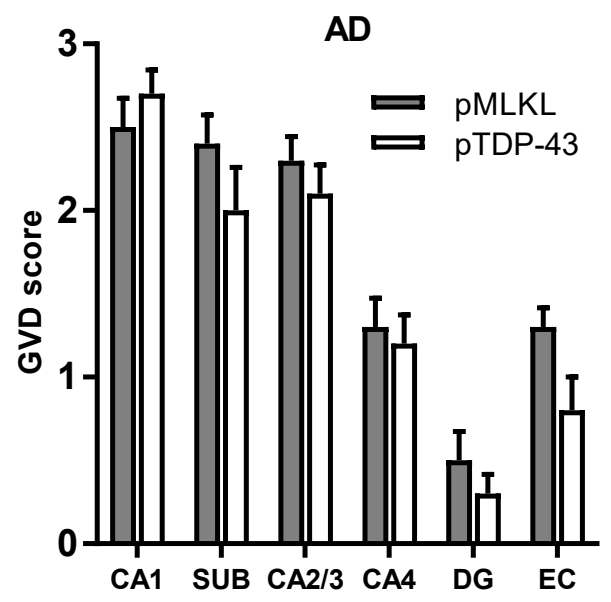

C

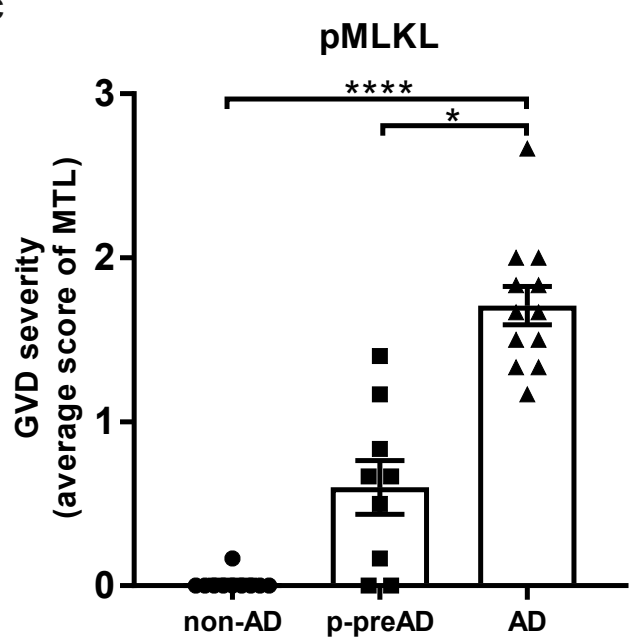

b

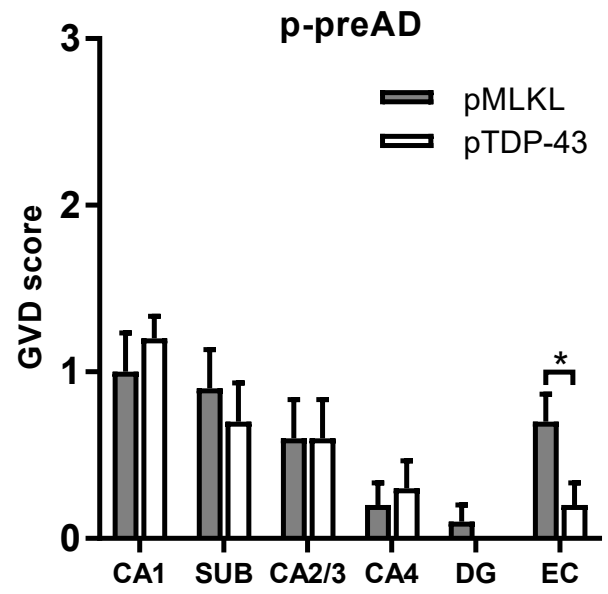

d

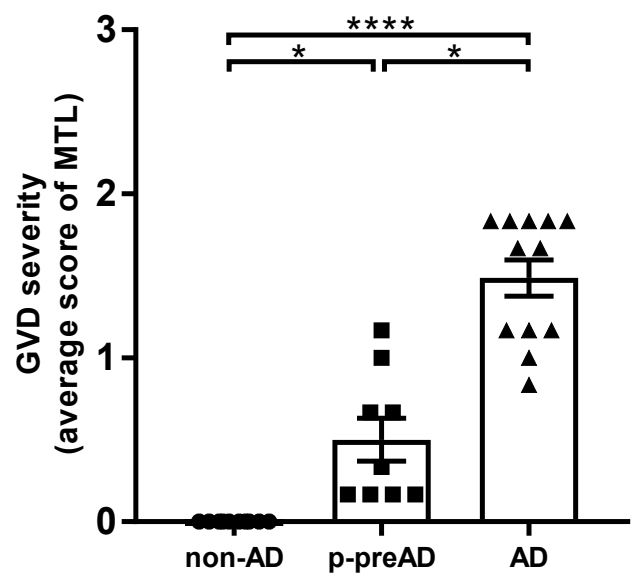


Suppl. Figure 5. Similarly to pMLKL-GVDn+ neurons, CK1ס-positive GVD neurons inversely correlate with neuronal density in the hippocampus and frontal cortex

a

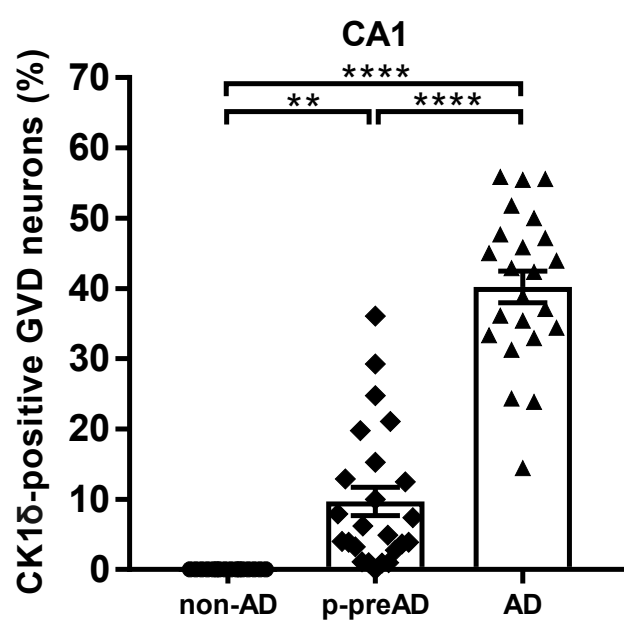

C

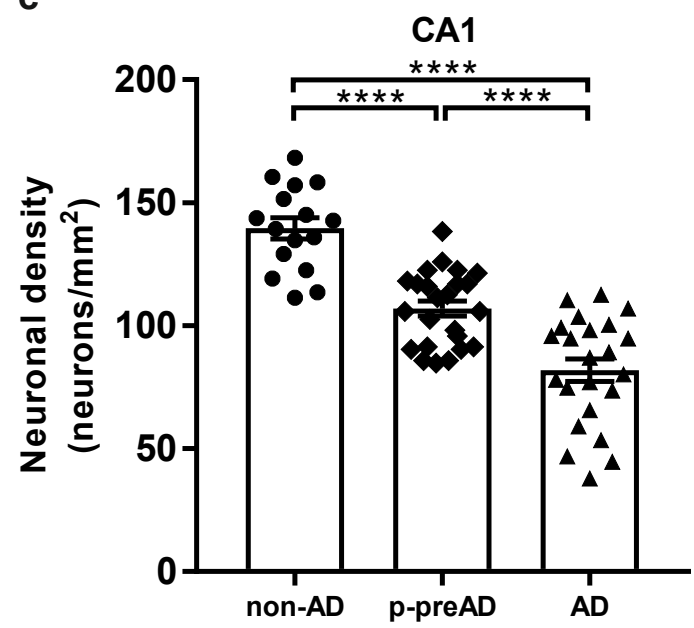

b

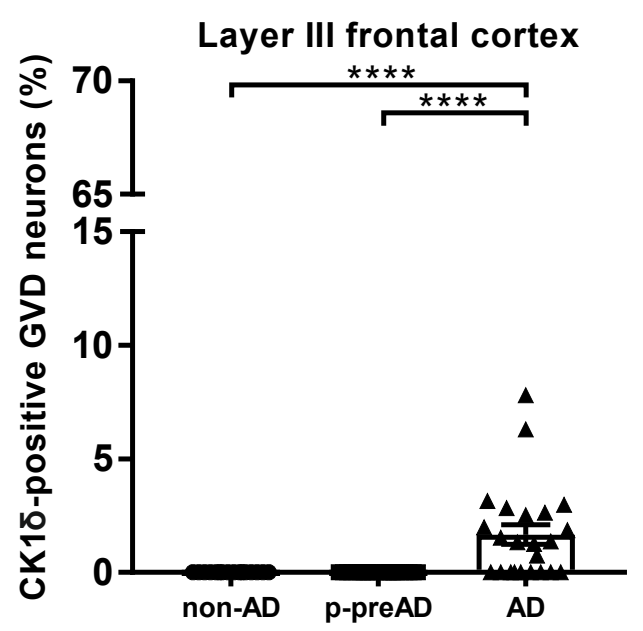

d

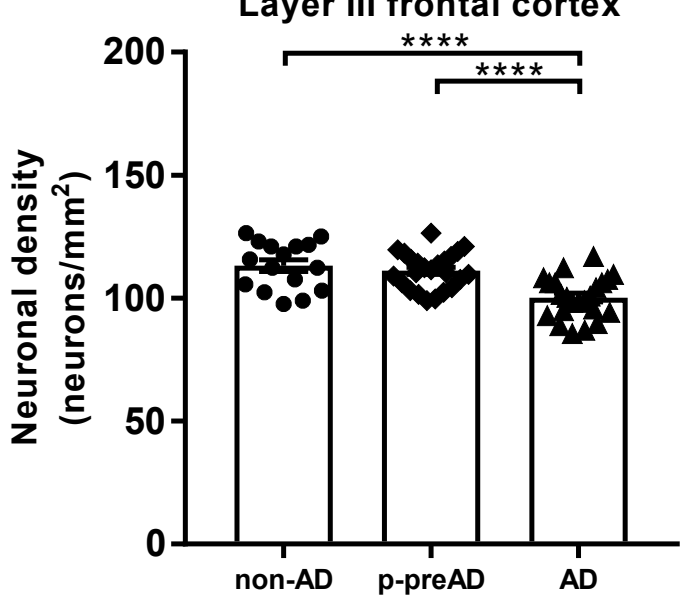


Suppl. Figure 6. Non-AD associated pMLKL-immunoreactive astrogliosis in CA4 and CA3 region of the hippocampus.

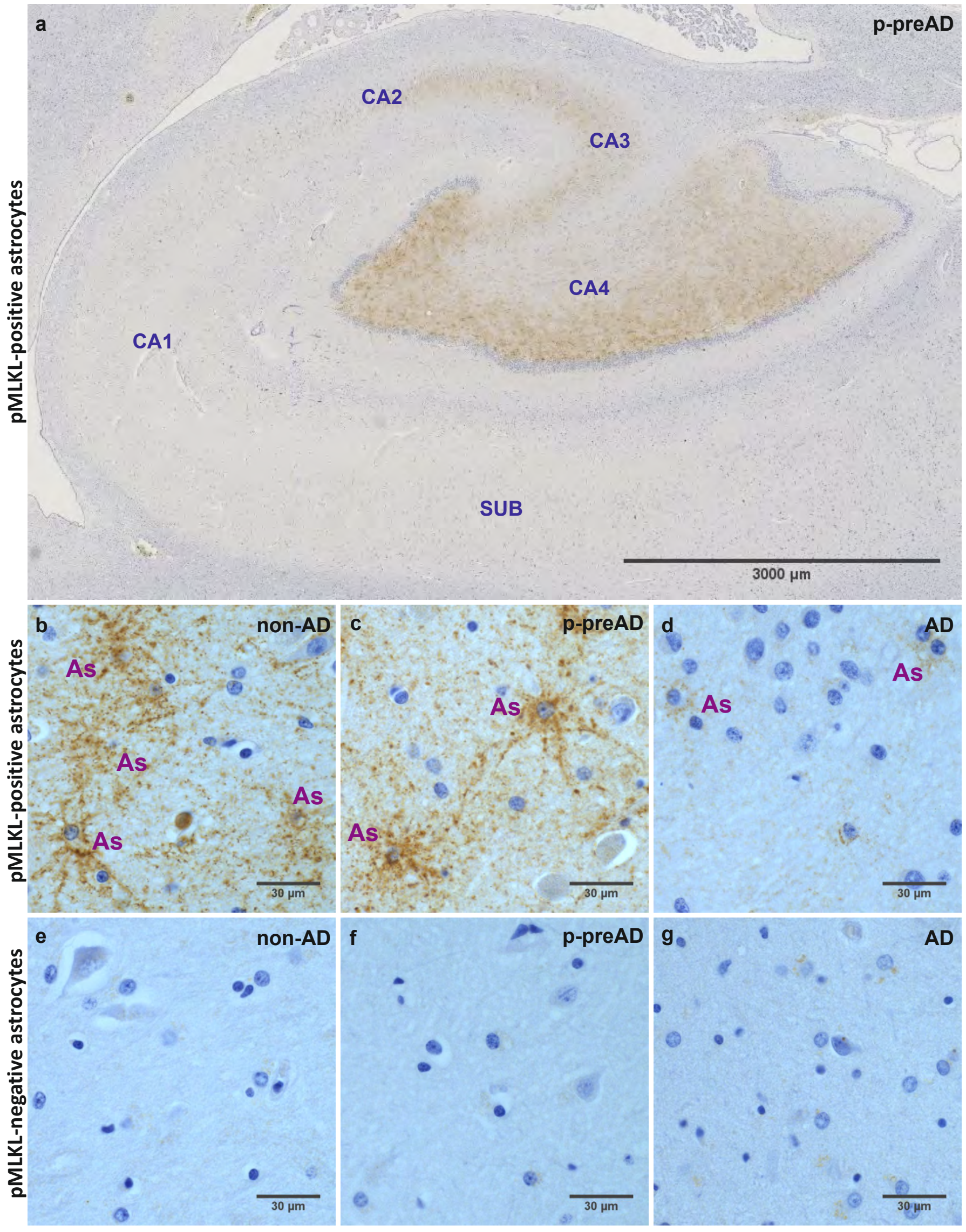




\section{Suppl. Figure 7. Full blots of all necroptosis-related proteins}

a

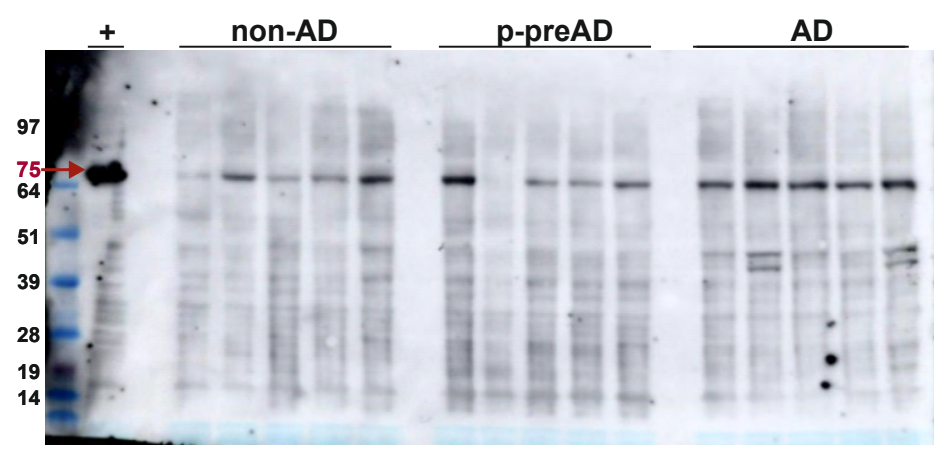

RIPK1

C

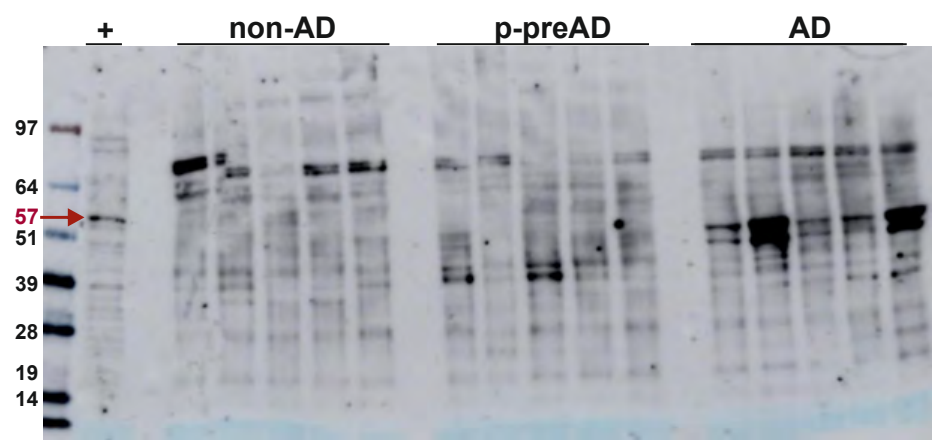

RIPK3

e

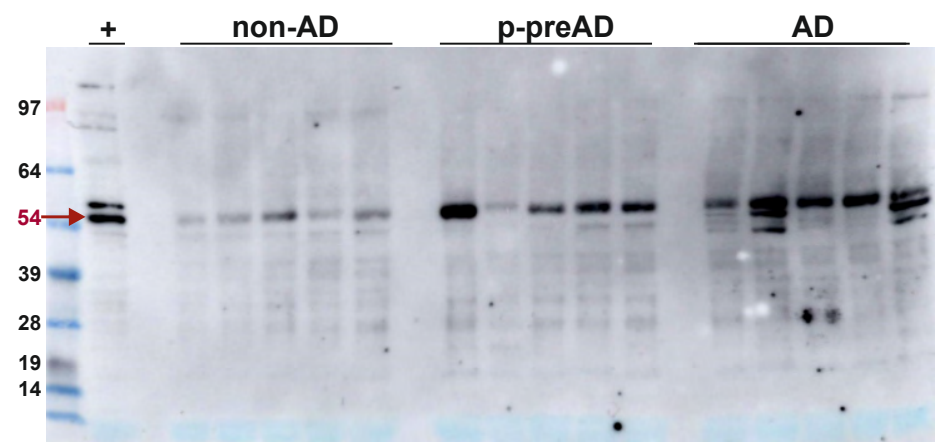

MLKL b

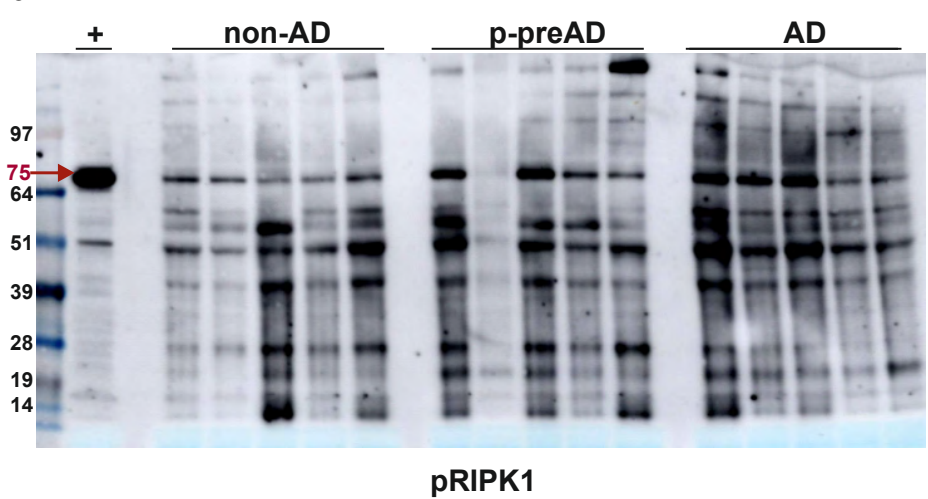

d

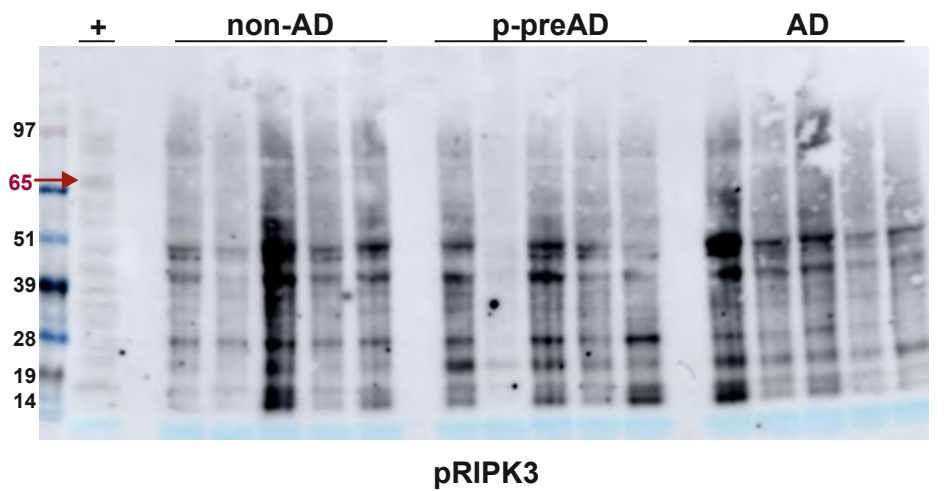

f

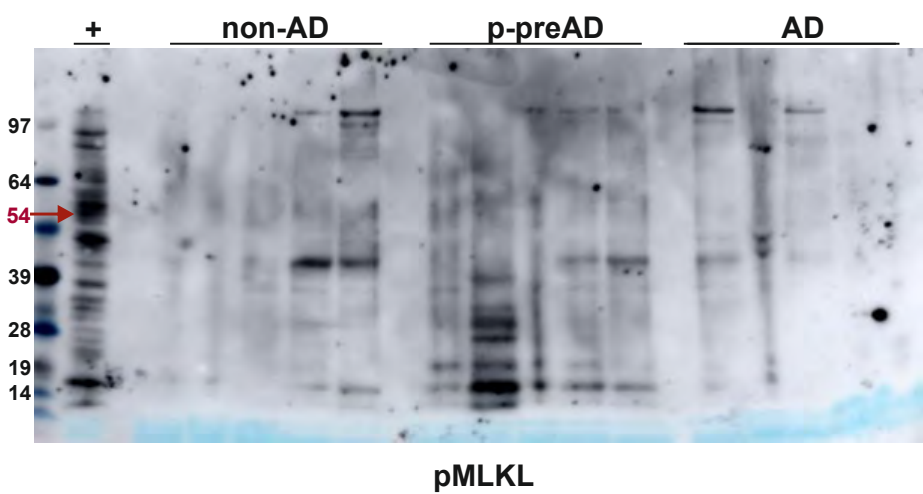


Table. 1. Cohort characteristics. The table shows age in years, sex, the ABMTL phase representing the distribution of $A \beta$ deposits in the subfields of the MTL [72], the stage of neurofibrillary tangle pathology according to Braak and Braak [9] (NFT stage), the CERAD score for the frequency of neuritic plaques according to Mirra et al. [43], the NIA-AA degree of AD pathology [30], the degree of dementia (CDR score) [44], the pMLKL-GVD stage, neuropathological diagnosis, and PMI. Abbreviations: $f=$ female; $m=$ male; $A D=$ Alzheimer's disease; $p$-pre $A D=$ preclinical $A D ; n o n-A D=$ non-demented control; $A D R P=$ Alzheimer's disease-related metabolic brain pattern; $A G D=$ argyrophilic grain disease, ARTAG $=$ aging-related tau astrogliopathy; $B=$ bleeding; $C A A=$ cerebrovascular angiopathy; $C D R=$ clinical dementia rating; $C E R A D=$ Consortium to Establish a Registry for Alzheimer's disease; $C M=$ carcinoma metastasis; $I=$ infarction; $M I=$ microinfraction; $M C I=$ mild cognitive impairment; $M T L=$ medial temporal lobe $;$ n.d. $=$ not determined $; N F T=$ neurofibrillary tangle; $N I A-A A=$ National Institute on Aging Alzheimer's Association; $P M I=$ post-mortem interval; $T$ = trauma.

\begin{tabular}{|c|c|c|c|c|c|c|c|c|c|c|}
\hline $\begin{array}{c}\text { Case } \\
\text { number }\end{array}$ & Age & Sex & $\begin{array}{l}\text { AßMTL } \\
\text { phase }\end{array}$ & $\begin{array}{l}\text { Braak NFT } \\
\text { stage }\end{array}$ & $\begin{array}{l}\text { CERAD } \\
\text { score }\end{array}$ & $\begin{array}{l}\text { NIA-AA } \\
\text { score }\end{array}$ & $\begin{array}{l}\text { CDR } \\
\text { score }\end{array}$ & $\begin{array}{l}\text { pMLKL- } \\
\text { GVD stage }\end{array}$ & $\begin{array}{c}\text { Neuropathological } \\
\text { diagnosis }\end{array}$ & PMI \\
\hline 1 & 87 & $\mathrm{~m}$ & 4 & 6 & 2 & 3 & 2 & 5 & $A D$ & 12 \\
\hline 2 & 69 & $\mathrm{~m}$ & 4 & 6 & 2 & 3 & 1 & 5 & $A D$ & 24 \\
\hline 3 & 57 & $\mathrm{~m}$ & 4 & 6 & 3 & 3 & 3 & 5 & $A D$ & 12 \\
\hline 4 & 55 & $f$ & 4 & 6 & 3 & 3 & 3 & 5 & $A D$ & 24 \\
\hline 5 & 74 & $f$ & 4 & 6 & 2 & 3 & n.a. & 5 & $A D$ & 72 \\
\hline 6 & 68 & $f$ & 4 & 6 & 2 & 3 & 1 & 5 & $A D, I$ & 120 \\
\hline 7 & 71 & $\mathrm{~m}$ & 4 & 6 & 2 & 3 & 2 & 5 & $A D, C A A, A R T A G, C M$ & 12 \\
\hline 8 & 74 & $\mathrm{~m}$ & 4 & 6 & 2 & 3 & 2 & 5 & $A D$ & 72 \\
\hline 9 & 76 & $\mathrm{~m}$ & 4 & 5 & 3 & 3 & 3 & 5 & $A D$ & 24 \\
\hline 10 & 81 & $f$ & 4 & 5 & 1 & 2 & 3 & 5 & $A D, C A A, I$ & 48 \\
\hline 11 & 83 & $\mathrm{~m}$ & 4 & 5 & 2 & 3 & 3 & 4 & $A D, C A A, I, B$ & 24 \\
\hline 12 & 89 & $f$ & 4 & 5 & 3 & 3 & 3 & 4 & $A D, C A A$ & 24 \\
\hline 13 & 78 & $f$ & 4 & 5 & 2 & 3 & 3 & 4 & $A D$ & 48 \\
\hline 14 & 71 & $\mathrm{~m}$ & 4 & 5 & 3 & 3 & 3 & 5 & $A D$ & 12 \\
\hline 15 & 76 & $\mathrm{~m}$ & 4 & 5 & 3 & 3 & 3 & 5 & $A D, A R T A G$ & 24 \\
\hline 16 & 78 & $f$ & 3 & 5 & 3 & 3 & 1 & 5 & $A D, B$ & 10 \\
\hline 17 & 83 & $\mathrm{~m}$ & 4 & 4 & 3 & 2 & 3 & 5 & $A D, I$ & 24 \\
\hline 18 & 89 & $f$ & 4 & 4 & 3 & 2 & 2 & 4 & $A D, C A A$ & 14 \\
\hline 19 & 65 & $\mathrm{~m}$ & 4 & 4 & 3 & 2 & 3 & 5 & $A D, T$ & 12 \\
\hline 20 & 82 & $\mathrm{~m}$ & 2 & 4 & 2 & 2 & 3 & 5 & $A D$ & 24 \\
\hline 21 & 82 & $\mathrm{~m}$ & 3 & 3 & 2 & 2 & 2 & 5 & $A D$ & 72 \\
\hline 22 & 98 & $f$ & 4 & 4 & 1 & 2 & 0.5 & 4 & $\mathrm{AD}, \mathrm{MCl}, \mathrm{I}$ & 24 \\
\hline 23 & 87 & $f$ & 4 & 4 & 1 & 2 & 3 & 4 & $A D, C A A, B$ & 24 \\
\hline 24 & 83 & $f$ & 3 & 4 & 1 & 2 & 0 & 4 & p-preAD, AGD, CM & 24 \\
\hline 25 & 85 & $f$ & 4 & 3 & 2 & 2 & 0 & 4 & p-preAD & 24 \\
\hline 26 & 74 & $\mathrm{~m}$ & 4 & 3 & 1 & 2 & 0 & 4 & p-preAD & 48 \\
\hline 27 & 74 & $\mathrm{~m}$ & 4 & 3 & 0 & 2 & n.a. & 3 & p-preAD & 96 \\
\hline 28 & 84 & $f$ & 3 & 3 & 0 & 2 & 0 & 3 & p-preAD, I & 96 \\
\hline 29 & 62 & $\mathrm{~m}$ & 4 & 3 & 0 & 2 & 0 & 4 & p-preAD & 23 \\
\hline 30 & 85 & $\mathrm{~m}$ & 2 & 3 & 1 & 1 & 0 & 3 & p-preAD, AGD & 44 \\
\hline 31 & 72 & $\mathrm{~m}$ & 2 & 3 & 0 & 1 & 0 & 4 & p-preAD, I & 72 \\
\hline
\end{tabular}




\begin{tabular}{|c|c|c|c|c|c|c|c|c|c|c|}
\hline 32 & 63 & $\mathrm{f}$ & 4 & 3 & 1 & 2 & 0 & 4 & p-preAD, I & 48 \\
\hline 33 & 83 & $f$ & 1 & 3 & 0 & 1 & 0 & 4 & p-preAD, AGD & 48 \\
\hline 34 & 83 & $\mathrm{f}$ & 3 & 3 & 1 & 2 & 0 & 4 & p-preAD & 72 \\
\hline 35 & 77 & $\mathrm{~m}$ & 1 & 2 & 0 & 1 & 0 & 3 & p-preAD & 24 \\
\hline 36 & 80 & $f$ & 3 & 2 & 0 & 1 & 1 & 1 & p-preAD, AGD & 12 \\
\hline 37 & 81 & $\mathrm{~m}$ & 3 & 2 & 0 & 1 & 2 & 0 & p-preAD, AGD, ADRP & 9 \\
\hline 38 & 75 & $\mathrm{~m}$ & 1 & 2 & 0 & 1 & 0 & 2 & p-preAD, AGD & 48 \\
\hline 39 & 77 & $f$ & 3 & 2 & 0 & 1 & 0 & 2 & p-preAD, I & 48 \\
\hline 40 & 66 & $f$ & 2 & 2 & 0 & 1 & n.a. & 2 & p-preAD & 48 \\
\hline 41 & 67 & $\mathrm{~m}$ & 3 & 2 & 0 & 1 & 0 & 2 & p-preAD & 24 \\
\hline 42 & 67 & $f$ & 2 & 2 & 0 & 1 & n.a. & 2 & p-preAD & 96 \\
\hline 43 & 73 & $f$ & 1 & 2 & 0 & 1 & 0 & 0 & p-preAD & 48 \\
\hline 44 & 71 & $\mathrm{~m}$ & 2 & 1 & 0 & 1 & 0 & 0 & p-preAD, CAA & 48 \\
\hline 45 & 65 & $\mathrm{~m}$ & 2 & 1 & 0 & 1 & 0 & 2 & p-preAD & 72 \\
\hline 46 & 84 & $f$ & 1 & 1 & 0 & 1 & 0 & 2 & p-preAD, AGD, I & 24 \\
\hline 47 & 64 & $\mathrm{~m}$ & 2 & 1 & 0 & 1 & 0 & 1 & p-preAD & 24 \\
\hline 48 & 46 & $\mathrm{~m}$ & 0 & 1 & 0 & 0 & 0 & 0 & Non-AD control & 29 \\
\hline 49 & 36 & $\mathrm{~m}$ & 0 & 0 & 0 & 0 & 0 & 0 & Non-AD control & 48 \\
\hline 50 & 66 & $f$ & 0 & 0 & 0 & 0 & 0 & 0 & Non-AD control, AGD & 48 \\
\hline 51 & 69 & $f$ & 0 & 0 & 0 & 0 & 0 & 0 & Non-AD control & 24 \\
\hline 52 & 61 & $\mathrm{~m}$ & 0 & 0 & 0 & 0 & 0 & 0 & Non-AD control, CM & 24 \\
\hline 53 & 61 & $\mathrm{~m}$ & 0 & 0 & 0 & 0 & 0 & 0 & Non-AD control & 24 \\
\hline 54 & 32 & $\mathrm{~m}$ & 0 & 0 & 0 & 0 & 0 & 0 & Non-AD control & n.a. \\
\hline 55 & 64 & $\mathrm{~m}$ & 0 & 0 & 0 & 0 & 1 & 0 & Non-AD control & 24 \\
\hline 56 & 67 & $\mathrm{~m}$ & 0 & 0 & 0 & 0 & 0 & 0 & Non-AD control & n.a. \\
\hline 57 & 60 & $\mathrm{~m}$ & 0 & 0 & 0 & 0 & 0 & 1 & Non-AD control, I & 24 \\
\hline 58 & 74 & $\mathrm{~m}$ & 0 & 0 & 0 & 0 & 0 & 0 & Non-AD control, CM, I, MI & 72 \\
\hline 59 & 62 & $\mathrm{~m}$ & 0 & 0 & 0 & 0 & 0 & 1 & Non-AD control & 48 \\
\hline 60 & 56 & $\mathrm{~m}$ & 0 & 0 & 0 & 0 & 0 & 0 & Non-AD control & 48 \\
\hline 61 & 45 & $\mathrm{~m}$ & 0 & 0 & 0 & 0 & 0 & 0 & Non-AD control & 24 \\
\hline 62 & 64 & $\mathrm{~m}$ & 0 & 0 & 0 & 0 & 0 & 0 & Non-AD control & n.a. \\
\hline 63 & 18 & $\mathrm{~m}$ & 0 & 0 & 0 & 0 & 0 & 0 & Non-AD control, $T$ & 24 \\
\hline
\end{tabular}

